\title{
Research Square \\ The Analysis of The Application and Mechanism of Traditional Chinese Medicine in Osteoarthritis Based on Data Mining and Network Pharmacology
}

Jie YANG

Orthopaedic Hospital https://orcid.org/0000-0001-7322-3166

Dijin JIAO

Orthopaedic Hospital

Guoguang Zhang

Liaoning University of Traditional Chinese Medicine

Juntong LIU

Liaoning University of Traditional Chinese Medicine

Chao QU

Liaoning University of Traditional Chinese Medicine

Hongxu CHEN

Liaoning University of Traditional Chinese Medicine

Chongmin CHEN

Orthopedic hospital

Yu SUN (D357602739@qq.com )

Orthopedic hospital https://orcid.org/0000-0002-2479-869X

\section{Research}

Keywords: Eucommiae Cortex, Achyranthis Bidentatae Radix, Osteoarthritis, Network Pharmacology, Molecular Docking

Posted Date: December 1st, 2020

DOI: https://doi.org/10.21203/rs.3.rs-113819/v1

License: (c) (i) This work is licensed under a Creative Commons Attribution 4.0 International License.

Read Full License 
The analysis of the application and mechanism of Traditional Chinese Medicine in osteoarthritis based on Data Mining and Network Pharmacology

YANG Jie ${ }^{1}$ JIAO Dijin ${ }^{1}$ ZHANG Guoguang ${ }^{2}$ LIU Juntong ${ }^{2}$ QU Chao ${ }^{2}$ CHEN Hongxu ${ }^{2}$ CHEN Chongmin ${ }^{1}$ SUN Yu $^{1 *}$

1. Shenyang Orthopedics Hospital, NO.115, Dongbei Road, Dadong District, Shenyang City, Liaoning Province, China

2. Liaoning Traditional Chinese Medicine University, NO.79 Chongshan Road,Shenyang City Liaoning Province, China Corresponding author: SUN Yu : 357602739@qq.com

Abstract: Background: Using Data Mining to retrieve the core drug of osteoarthritis in clinic , predicting the drug molecular action target through the Network Pharmacology, combining with the related targets of osteoarthritis to identify the key nodes of the interaction, exploring the pharmacological mechanism of Traditional Chinese Medicine against osteoarthritis and other possible mechanisms of actions. Methods: Pubmed, CNKI, VIP, CBM and WanFang Database was used to retrieve the commonly used therapeutic formulations for osteoarthritis patients in clinical, and screen out the core drugs through the Ancient and Modern Medical Case Cloud Platform and software Gephi, filtered out the core drug molecules and targets combined with TCMSP database and the targets of osteoarthritis in Genecard, OMIM database, impoting those datas into R project and Cytoscape to construct the intersection model of Drug molecule-osteoarthritis, carrying out PPI network and GO and KEGG enrichment analysis with String database. Vina molecular docking was implemented to draw molecular docking diagram, and the results were analyzed after comprehensive analysis. Results: The core drug pairs were identified as "Eucommiae Cortex - Achyranthis Bidentatae Radix" through correlation analysis, complex network analysis basing on the coefficient. "Eucommiae Cortex Achyranthis Bidentatae Radix" can intervene cell behaviors through multiple pathways and regulate cell metabolism, cytokine synthesis, oxidative , cellular immunity as a consequence of topology analysis in String Database. Conclusions: "Eucommia bark - achyranthes" drug molecules can be combined with the target to produce hydrogen bond, hydrophobic function and Pi-Pi directly or indirectly affecting the corresponding targets, to participate in the regulation of osteogenesis and osteoclast proliferation, protect the extracellular matrix, inhibition of cell apoptosis and anti-inflammatory for resistance to osteoarthritis, also, providing the basis for interpretation of its action mechanism.

Key Words: Eucommiae Cortex, Achyranthis Bidentatae Radix, Osteoarthritis, Network Pharmacology, Molecular Docking

Background: Osteoarthritis is a common form of irreversible degenerative joint disease, with clinical manifestations of pain, movement disorders, joint deformity, which has seriously affected the quality of people's daily life. The current treatment Methodss are mostly sports physical therapy, oral medication, acupuncture, massage, joint cavity injection, and even orthotics, joint replacement, etc.[1]. The pathological basis of osteoarthritis is considered to be chondrocyte apoptosis and the degeneration of extracellular matrix (ECM) changes[2], articular cartilage occupies about 1-5\% of the total bone volume and cartilage cells can recognize and respond to mechanical stimuli which makes itself to a pressure-bearing tissue[3]. Chondrocytes can be simultaneously synthesized aggrecan (ACAN) as the main component of the extracellular matrix[4], and collagen II constitute the extracellular matrix, in turn, provide the basis for the necessary environment to normal chondrocyte function. In addition, Synovium, another important tissue in the joint, can be divided into synovial macrophages and synovial fibroblasts, Its main function is to secrete joint synovial fluid, provide nutrients for cartilage cells and material- exchanging. The abnormal performance of these tissues can lead to the occurrence and 
development of osteoarthritis, and the normal performance of its function may be related to exosomes[5]. This research is to use data mining technology to collect prescriptions commonly used in the treatment of osteoarthritis, analyze the composition of the drugs, and screen out the core drugs to interpret the mechanism of action and association between drugs and diseases through network pharmacology and molecular docking Methodss, and predict other possible mechanisms of action.

\section{Materials and Methods}

\subsection{Literature sources}

Search CNKI, VIP, CBM, Pubmed, WanFang Database with "osteoarthritis", "degenerative arthritis", "Bizheng", "Gubi syndrome", "Chinese medicine" as the subject terms, starting from January 2010 to June 2020. A total of 3,319 duplicate documents were excluded, and a total of 207 clinical observations, case analyses, medical record summaries, master and doctoral dissertations on the treatment of osteoarthritis with Traditional Chinese Medicine were screened out. The data involved were extracted by two independent personnel.

\subsection{Inclusion criteria}

a. Literatures that met the diagnostic criteria for osteoarthritis. b. Using Chinese herbal compound preparations as intervention measures. c. The test subject must be clinical patients. d. Experiment design and statistical Methodss were scientific and effective. e. The same prescription, the same author has more than 2 articles, only 1 article was included. f. There must be a clear prescription and drug dosage in the literature.

\subsection{Exclusion criteria}

a. Literature review. b. Animal experiment literature. c. There is no literature with clear prescription and dosage. d. Negative results. e. Missing clinical data. f. Basic scientific research.

\subsection{Data extraction}

Input the data within the literature into the EXCEL table and saved and imported Ancient and Modern Medical Record Cloud Platform (http://www.yiankb.com/). The association rule of analysis, drug frequency statistics, complex network construction and etc. were performed to analyze the drug compatibility law, and the pair of drug combination with confidence level $>0.9$ and support level $>0.2$ was selected as the core drug compatibility.

\subsection{Network pharmacology}

Traditional Chinese Medicine Systems Pharmacology Database and Analysis Platform (TCMSP, http://tcmspw.com/tcmsp.php)were using to search the chemical components and its targets of core Chinese medicine which was selected in the previous step with the filter condition $\mathrm{OB} \geq 30 \%$. Then, got the gene targets of diseases through databases such as Genecards, GEO, OMIM, and used "Osteoarthritis, Arthritis, Degenerative" as search terms. After taking the intersection of the compound target contained in the drug and the disease gene target, imported Cytoscape 3.6.0 software to construct a "compound-target" network. Construction signaling pathway targets the core screened by STRING internet PPI protein interaction network, using R Project for KEGG GO annotation enrichment analysis and understanding of the core target.

\subsection{Molecular docking}

Searched the 3D structure diagram of the core target in the RSCB database and the 2D structure diagram of the compound through the PubChem database, using Chem 3D software to make it into a 3D structure diagram, and utilizing PyMOL, Autodock, Discovery Studio software to hydrogenate, dewatering, de-impurity and other preprocessing with the core target, whereafter, the Vina docking was performed, and the conformational map of the molecule with the lowest binding energy value was 
docked with the 3D map of the protein. Ligplus was used to display the hydrogen bond distance and hydrophobicity of the drug molecule and the surrounding residues.

\section{Results}

\subsection{Drug frequency}

According to the inclusion and exclusion criteria, a total of 3,292 articles were retrieved from various databases, and 184 articles were finally chosen and included in the study, including 94 prescriptions and 194 Traditional Chinese Medicines. The frequency statistics of the Chinese medicines contained in the prescriptions were performed, and 17 with a frequency of $\geq 45$, which the top 5 were Achyranthes bidentata, Angelica, Licorice, Eucommia, Chuanxiong, (Table 1).

Table 1. Traditional Chinese Medicine Frequency

\begin{tabular}{|c|c|c|c|c|}
\hline Num & $\begin{array}{c}\text { Drug } \\
\text { (Latin name) }\end{array}$ & Frequency & Percentage & Average dose $(\mathrm{g})$ \\
\hline 1 & Achyranthis Bidentatae Radix & 163 & $77.25 \%$ & 13.56 \\
\hline 2 & Angelicae Sinensis Radix & 142 & $67.30 \%$ & 13.04 \\
\hline 3 & licorice & 92 & $43.60 \%$ & 6.95 \\
\hline 4 & Eucommiae Cortex & 90 & $42.65 \%$ & 14.42 \\
\hline 5 & Angelicae Biseratae Radix & 88 & $41.71 \%$ & 13.18 \\
\hline 6 & Chuanxiong Rhizoma & 87 & $41.23 \%$ & 11.84 \\
\hline 7 & Rehmanniae Radix Praeparata & 86 & $40.76 \%$ & 17.54 \\
\hline 8 & Paeoniae Radix Alba & 83 & $39.34 \%$ & 15.42 \\
\hline 9 & Herba Taxilli & 80 & $37.91 \%$ & 16.64 \\
\hline 10 & Gentiana Macrophylla Pall & 72 & $34.12 \%$ & 12.25 \\
\hline 11 & Poria $\operatorname{Cocos}$ (Schw.) Wolf. & 71 & $33.65 \%$ & 14.48 \\
\hline 12 & Saposhnikoviae Radix & 60 & $28.44 \%$ & 11.07 \\
\hline 13 & Hedysarum Multijugum Maxim. & 55 & $26.07 \%$ & 23.85 \\
\hline 14 & Carthami Flos & 54 & $25.59 \%$ & 9.59 \\
\hline 15 & Radix Clematidis & 53 & $25.12 \%$ & 15.13 \\
\hline 16 & Drynariae Rhizoma & 50 & $23.70 \%$ & 15.14 \\
\hline 17 & Chaenomeles Sinensis (Thouin) Koehne & 46 & $21.80 \%$ & 12.83 \\
\hline
\end{tabular}

\subsection{Association rule analysis}

Perform compatibility correlation analysis on the drugs in the included prescriptions, and screen matching drug combinations based on confidence, support, and lift (lift $>1$ indicates that there is a positive correlation between paired drugs). Visually drug displays combinations in Gephi-0.9.2 with co-occurrence frequency $\geq 50$, confidence $>0.8$, support $>0.25$. (Table 2, Fig 1).

Table 2. Drug association analysis

\begin{tabular}{|c|c|c|c|c|c|}
\hline Drug A & Drug B & $\begin{array}{c}\text { Co-occurrence } \\
\text { Frequency }\end{array}$ & Confidence & Support & Lift \\
\hline Angelicae Sinensis Radix & Achyranthis Bidentatae Radix & 113 & 0.8 & 0.54 & 1.04 \\
\hline Eucommiae Cortex & Achyranthis Bidentatae Radix & 82 & 0.91 & 0.39 & 1.18 \\
\hline Angelicae Biseratae Radix & Achyranthis Bidentatae Radix & 79 & 0.9 & 0.37 & 1.17 \\
\hline Chuanxiong Rhizoma & Angelicae Sinensis Radix & 76 & 0.87 & 0.36 & 1.29 \\
\hline licorice & Achyranthis Bidentatae Radix & 76 & 0.83 & 0.36 & 1.07 \\
\hline Chuanxiong Rhizoma & Achyranthis Bidentatae Radix & 72 & 0.83 & 0.34 & 1.07 \\
\hline Herba Taxilli & Achyranthis Bidentatae Radix & 72 & 0.9 & 0.34 & 1.17 \\
\hline
\end{tabular}




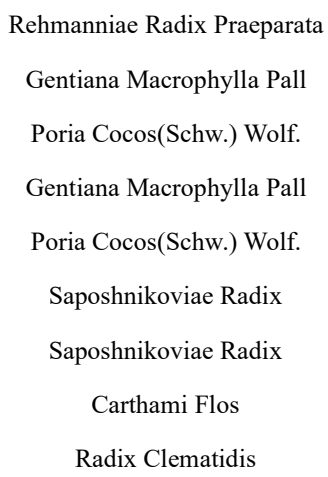

Asari Radix Et Rhizoma

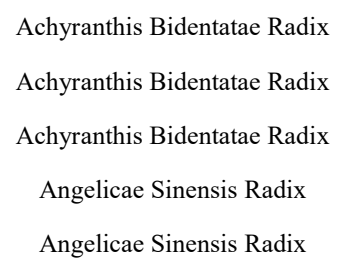

Achyranthis Bidentatae Radix

$\begin{array}{llll}70 & 0.81 & 0.33 & 1.05 \\ 61 & 0.85 & 0.29 & 1.1 \\ 60 & 0.85 & 0.28 & 1.1 \\ 59 & 0.82 & 0.28 & 1.22 \\ 58 & 0.82 & 0.27 & 1.22 \\ 50 & 0.83 & 0.24 & 1.07 \\ 49 & 0.82 & 0.23 & 1.22 \\ 47 & 0.87 & 0.22 & 1.13 \\ 47 & 0.89 & 0.22 & 1.15 \\ 44 & 0.96 & 0.21 & 1.24\end{array}$

\subsection{Complex network analysis}

Import data from correlation analysis into Gephi-0.9.2 for statistics, calculate topological characteristic parameters "Weighted degree", "Harmonic closeness centrality", "Eigen centrality", "Betweeness centrality", ect. The average node degree of the network is 2.429 , and the graph density is 0.187. Achyranthis Bidentatae Radix, Angelicae Sinensis Radix, Chuanxiong Rhizoma, Gentiana Macrophylla Pall ranked top among them. (Table 3).

Table 3. Complex network topology value

\begin{tabular}{cccccc}
\hline Num & Drug & $\begin{array}{c}\text { Weighted } \\
\text { degree }\end{array}$ & $\begin{array}{c}\text { Harmonic closeness } \\
\text { centrality }\end{array}$ & $\begin{array}{c}\text { Eigen } \\
\text { centrality }\end{array}$ & $\begin{array}{c}\text { Betweeness } \\
\text { centrality }\end{array}$ \\
\hline 1 & Achyranthis Bidentatae Radix & 13 & 1 & 1 & 0.910256 \\
2 & Angelicae Sinensis Radix & 5 & 0.692308 & 0.618248 & 0.038462 \\
3 & Chuanxiong Rhizoma & 2 & 0.576923 & 0.39075 & 0 \\
4 & Gentiana Macrophylla Pall & 2 & 0.576923 & 0.39075 & 0 \\
5 & Poria Cocos(Schw.) Wolf. & 2 & 0.576923 & 0.39075 & 0 \\
6 & Saposhnikoviae Radix & 2 & 0.576923 & 0.39075 & 0 \\
7 & Eucommiae Cortex & 1 & 0.538462 & 0.242832 & 0 \\
8 & Angelicae Biseratae Radix & 1 & 0.538462 & 0.242832 & 0 \\
9 & licorice & 1 & 0.538462 & 0.242832 & 0 \\
10 & Herba Taxilli & 1 & 0.538462 & 0.242832 & 0 \\
11 & Rehmanniae Radix Praeparata & 1 & 0.538462 & 0.242832 & 0 \\
12 & Carthami Flos & 1 & 0.538462 & 0.242832 & 0 \\
13 & Radix Clematidis & 1 & 0.538462 & 0.242832 & 0 \\
14 & Asari Radix Et Rhizoma & 1 & 0.538462 & 0.242832 & 0 \\
\hline
\end{tabular}

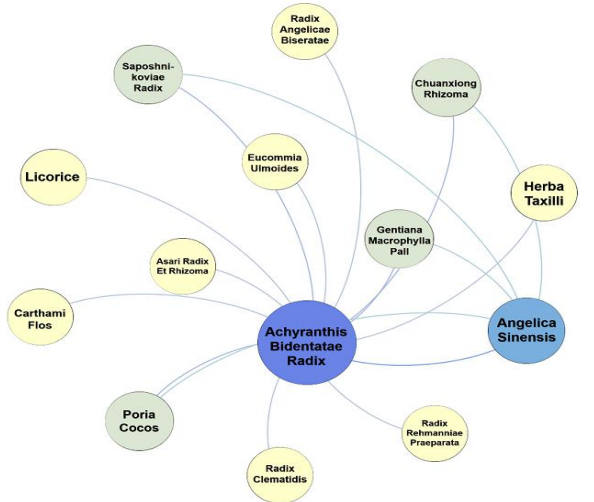

Fig 1. Association Analysis
In summary, the results of frequency analysis, correlation analysis, and complex network analysis can prompt "Achyranthis Bidentatae Radix-Angelicae Sinensis Radix", "Eucommiae Cortex-Achyranthis Bidentatae Radix", "Angelicae Biseratae Radix -Achyranthis Bidentatae Radix" as compatibility of core drugs for the treatment of osteoarthritis, The confidence of "Eucommiae Cortex-Achyranthis Bidentatae Radix" drug pair is 0.91, so this study selects "Achyranthis Bidentatae Radix-Eucommiae Cortex" drug pair for 
Network Network Pharmacology analysis and molecular mechanism discussion.

2.4 Discussion on Molecular Mechanism of Core Compatibility

2.4.1 "Achyranthis Bidentatae Radix-Angelicae Sinensis Radix" composition -target network construction

With oral bioavailability rate $(\mathrm{OB}) \geq 30 \%$ and drug-like property $(\mathrm{DL}) \geq 0.18$ as the screening conditions, 48 pharmaceutical compound components and 211 targets are retrieved on the TCMSP platform. Using Genecard and OMIM database with "Arthritis, Degenerative" and "Osteoarthritis" as the subject terms, 5892 targets are retrieved in total, removing the duplicate targets, 3737 targets and chemical composition targets are intersected and 156 intersecting targets are obtained, (Fig 3), Build a complex network of "Achyranthis Bidentatae Radix-Eucommiae Cortex" compounds and disease targets in Cytoscape, (Fig 4), The Degree $\geq 10$, Betweenness, and Closeness was used as the screening basis, and finally 14 compound components and 8 targets were obtained, Table 4 . The top 5 compounds: (Fig.2)

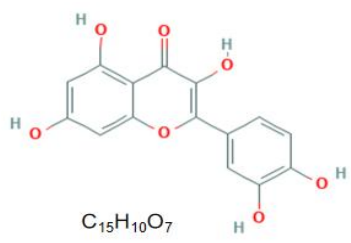

Fig.2

a. Quercetin

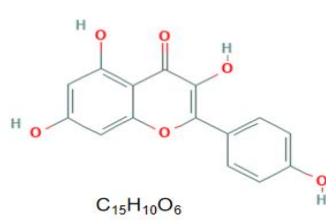

b. Kaempferol

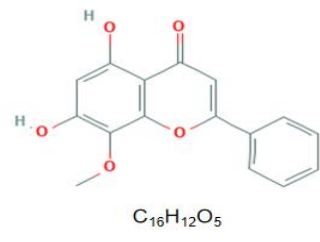

c. Wogonin

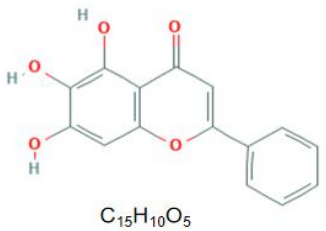

d. Baicalein

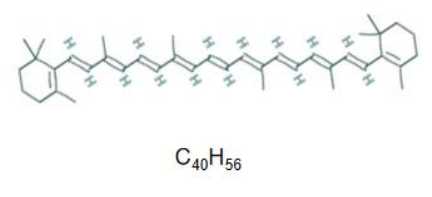

e. $\beta$-carotene

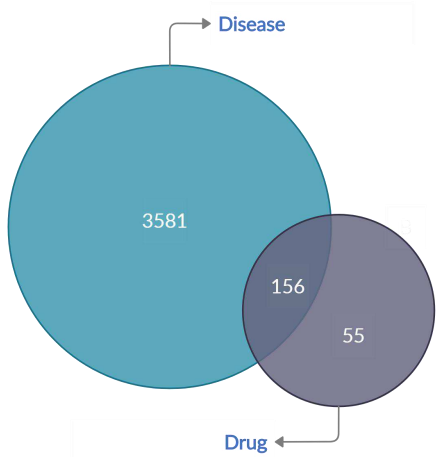

Fig 3. Venn diagram

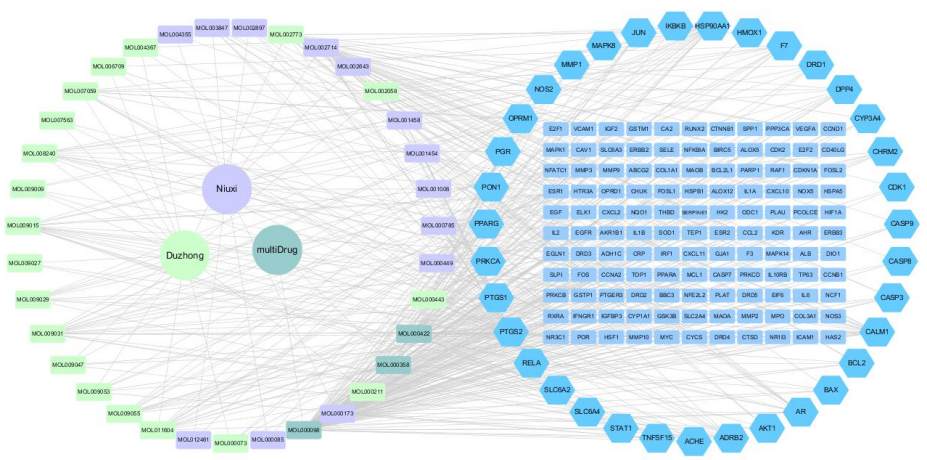

Fig 4. Compound and component target network

Table 4. Compound and ingredient targets (Degree $\geq 10$ )

\begin{tabular}{cccccc}
\hline Num & Name & Compound / Ingredient target & Degree & Betweenness & Closeness \\
\hline 1 & MOL000098 & Quercetin & 111 & 0.63397326 & 0.58282209 \\
2 & MOL000422 & Kaempferol & 41 & 0.14182446 & 0.4185022 \\
3 & MOL000173 & Wogonin & 35 & 0.09817351 & 0.39748954 \\
4 & PTGS2 & PTGS2 & 28 & 0.11484317 & 0.52631579 \\
5 & MOL002714 & Baicalein & 28 & 0.09290706 & 0.38617886 \\
6 & HSP90AA1 & HSP90AA1 & 20 & 0.05689962 & 0.48843188
\end{tabular}




\begin{tabular}{|c|c|c|c|c|c|}
\hline 7 & MOL002773 & Beta-carotene & 20 & 0.05118076 & 0.37109375 \\
\hline 8 & CALM1 & CALM1 & 18 & 0.02350805 & 0.36893204 \\
\hline 9 & MOL009031 & Cinchonan-9-al, 6'-methoxy-, (9R)- & 18 & 0.04400867 & 0.36821705 \\
\hline 10 & PTGS1 & PTGS1 & 17 & 0.05278235 & 0.4859335 \\
\hline 11 & MOL000358 & Beta-sitosterol & 17 & 0.03652413 & 0.37549407 \\
\hline 12 & MOL009015 & (-)-Tabernemontanine & 17 & 0.04011822 & 0.36964981 \\
\hline 13 & MOL011604 & Syringetin & 14 & 0.01432355 & 0.36538462 \\
\hline 14 & MOL000449 & Stigmasterol & 13 & 0.04099078 & 0.36964981 \\
\hline 15 & $\mathrm{AR}$ & $\mathrm{AR}$ & 12 & 0.04609877 & 0.46454768 \\
\hline 16 & MOL008240 & $\begin{array}{l}\text { (E)-3-[4-[(1R,2R)-2-hydroxy-2-(4-hydro } \\
\text { xy-3-methoxy-phenyl)-1-methylol-ethox } \\
\text { y]-3-methoxy-phenyl]acrolein }\end{array}$ & 12 & 0.00912432 & 0.35984848 \\
\hline 17 & MOL000785 & palmatine & 12 & 0.00872853 & 0.36259542 \\
\hline 18 & ADRB2 & ADRB2 & 11 & 0.02606931 & 0.44496487 \\
\hline 19 & ESR1 & ESR1 & 11 & 0.00288936 & 0.29733959 \\
\hline 20 & MOL009055 & hirsutin_qt & 11 & 0.01288744 & 0.35849057 \\
\hline 21 & F7 & F7 & 10 & 0.01665942 & 0.42128603 \\
\hline 22 & MOL007059 & 3-beta-Hydroxymethyllenetanshiquinone & 10 & 0.01156619 & 0.35714286 \\
\hline
\end{tabular}

\subsubsection{PPI network construction}

Import the above targets into the STRING database, construct the PPI network diagram, and output the tsv table at the same time, and import the key nodes into the Cytoscape using the Cytohubba plug-in. The color more darker is, the more important its position in the network, (Fig 5-6), The Fig suggests that ESR1, PTGS2, AR, HSP90AA1, CALM1, and PTGS1 are the core targets of drug action.

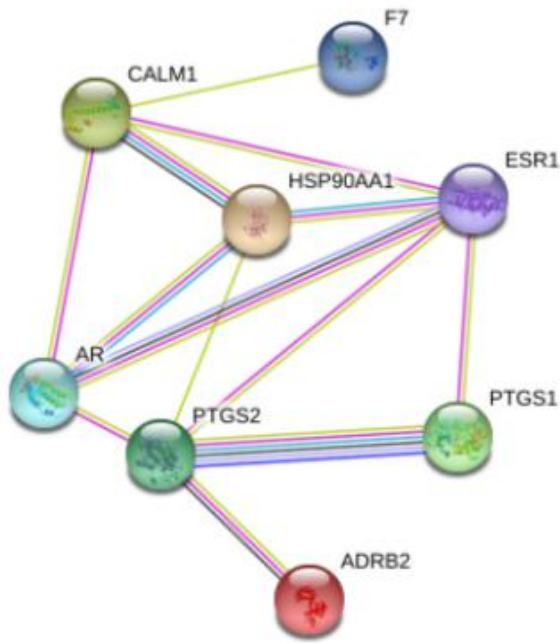

Fig 5. PPI network

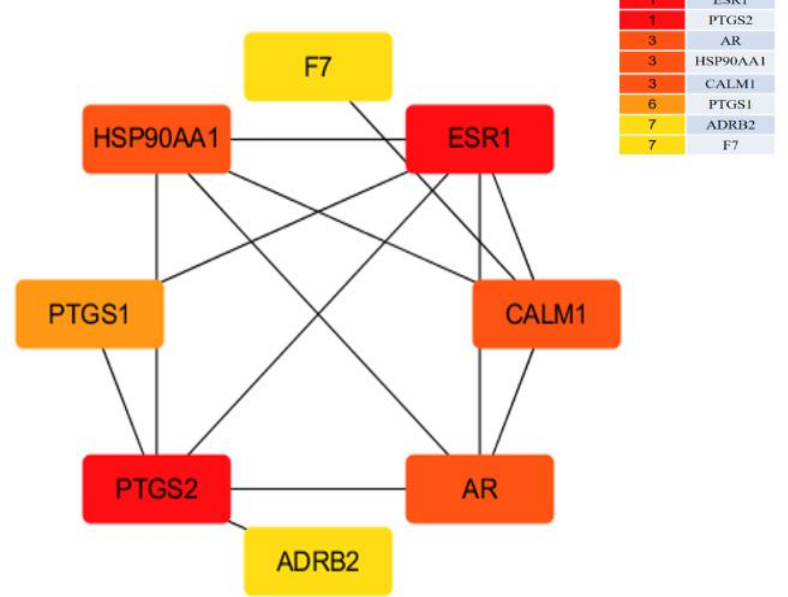

Fig 6. Cytohubba Visualization

\subsubsection{GO annotation}

Import the above 8 differential genes into R Project and use the BiocManager package for GO annotation, Use the STRING database to expand its genes and find its pre-gene or cross-linked genes due to the incomplete information of $\mathrm{CC}$ (Cellular Component) in GO annotations is impossible to locate the differential genes in cells. The expanded gene is F3, STIP1, HSPA8, NCOA2, CDC37. BP (Biological Process) $p<0.05$ for the number of 291, CC (Cellular Component) $p<0.05$ for the number of 17, MF (Molecular Function) $p<0.05$ for the number of 65, (Fig 7-9). 


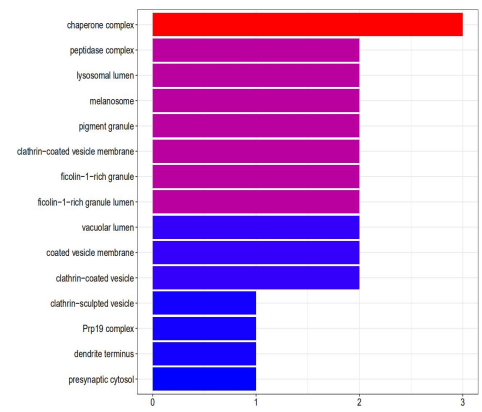

Fig 7. GO-CC Bar graph

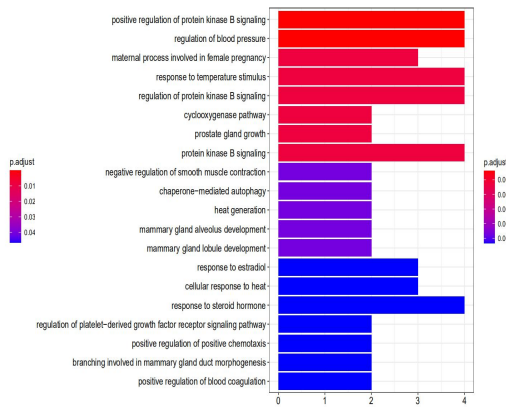

Fig 8. GO-BP Bar graph

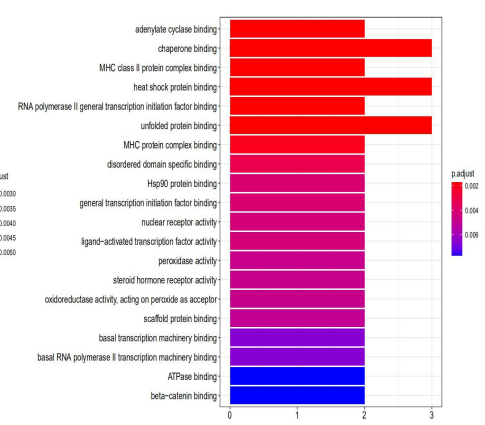

Fig 9. GO-MF Bar graph

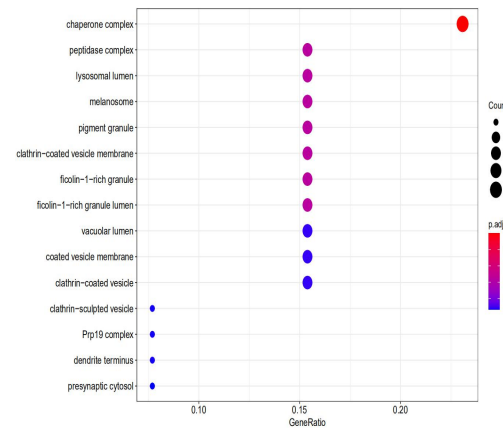

Fig 7a. GO-CC Bubble

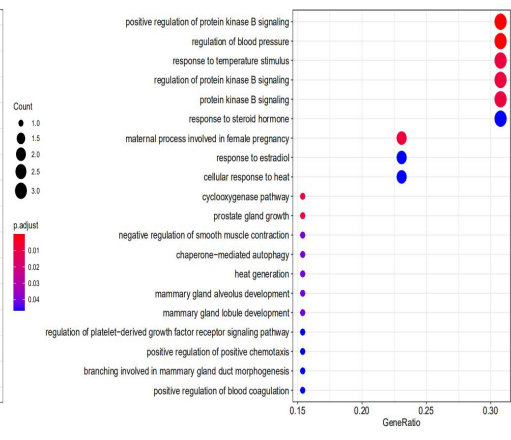

Fig 8a. GO-BP Bubble

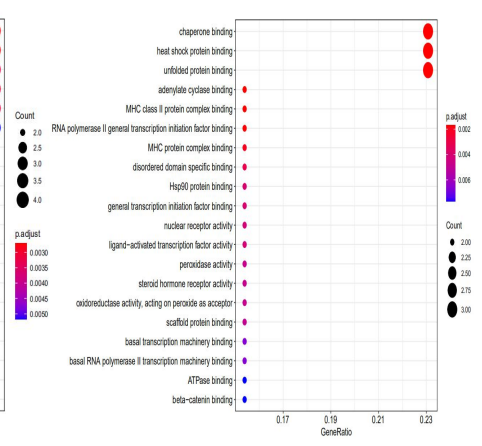

Fig 9a. GO-MF Bubble

In the Fig, the abscissa is the GO enrichment number and the ordinate is the GO name. The closer the color is to red, the higher the significance and the smaller the $p$ value.

\subsubsection{KEGG pathway analysis}

Performing KEGG pathway of 8 genes above by making use of BioManager package in R Project which can be infer the main functional pathway contained Regulation of lipolysis in adipocytes (hsa04923), Estrogen signaling pathway (hsa04915), Arachidonic acid metabolism (hsa00590), Renin secretion (hsa04924), IL-17 signaling pathway (hsa04657) ect. (Fig 10-11).

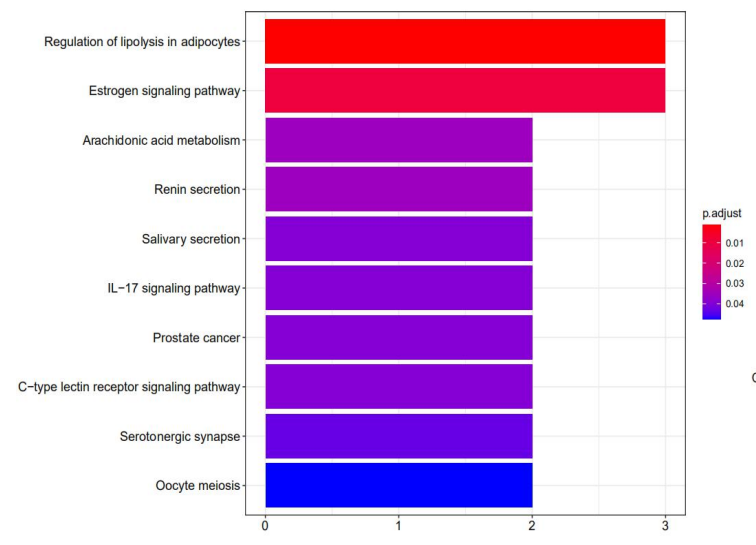

Fig 10. KEGG Bar graph

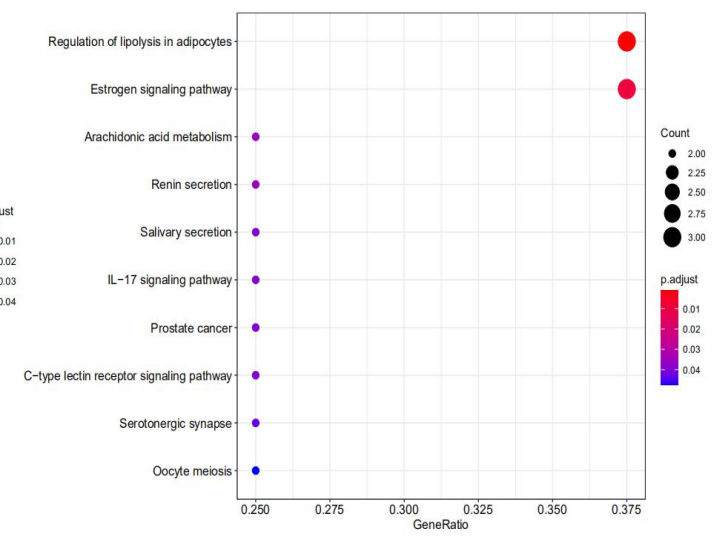

Fig 10-1. KEGG Bubble

In the Fig, the abscissa is the KEGG enrichment ratio, the ordinate is the KEGG name, and the size of the circle is the enrichment number. The closer the color is to red, the higher the significance of the enrichment and the smaller the p value.

2.4.5 Molecular docking of compound and target

Perform vina docking of the top 5 compounds in Degree with the 6 core targets, and calculate their binding energy, (Table 5), draw a heat map based on the binding energy, the higher the absolute value 


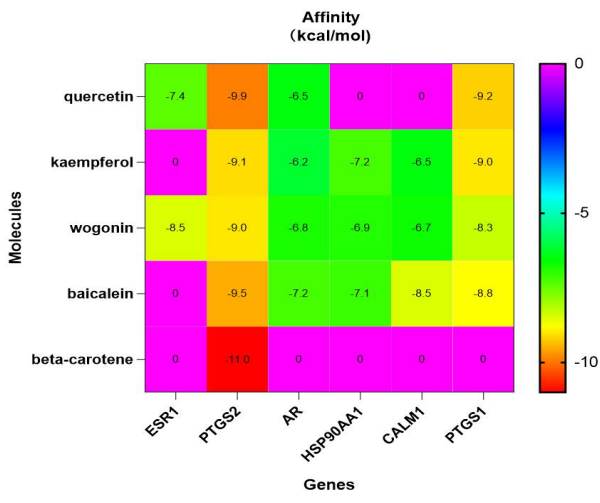

Fig 11. Affinity heat map

of affinity, the tighter the molecular binding(Fig 11). Do molecular docking of molecules with absolute binding energy greater than 7.0 and proteins and draw docking pattern diagrams. Further observation of the spatial structure, binding residues, hydrogen bond distance, hydrophilicity and hydrophobicity between molecules and proteins through 2D and 3D model diagrams. From the Fig, it can be found that PTGS1 and PTGS2 bind the most compound molecules to protein molecules. The molecular docking diagram is as shown in Fig 12-13.

Table 5. Affinity of compound to core target

\begin{tabular}{|c|c|c|c|c|c|c|}
\hline $\begin{array}{c}\text { Compound } \\
\text { number }\end{array}$ & $\begin{array}{c}\text { Compound } \\
\text { name }\end{array}$ & $\begin{array}{l}\text { Chemical } \\
\text { formula }\end{array}$ & $\begin{array}{l}\text { Relative } \\
\text { molecular } \\
\text { weight }\end{array}$ & CAS & $\begin{array}{l}\text { Binding } \\
\text { protein }\end{array}$ & $\begin{array}{c}\text { Affinity } \\
\text { (kcal/mol) }\end{array}$ \\
\hline \multirow{4}{*}{ MOL000098 } & \multirow{4}{*}{ Quercetin } & \multirow{4}{*}{$\mathrm{C}_{15} \mathrm{H}_{10} \mathrm{O}_{7}$} & \multirow{4}{*}{302.25} & \multirow{4}{*}{$73123-10-1$} & HSP90AA1 & -7.4 \\
\hline & & & & & PTGS1 & -9.2 \\
\hline & & & & & PTGS2 & -9.9 \\
\hline & & & & & AR & -6.5 \\
\hline \multirow{5}{*}{ MOL000422 } & \multirow{5}{*}{ Kaempferol } & \multirow{5}{*}{$\mathrm{C}_{15} \mathrm{H}_{10} \mathrm{O}_{6}$} & \multirow{5}{*}{286.25} & \multirow{5}{*}{$520-18-3$} & HSP90AA1 & -7.2 \\
\hline & & & & & PTGS1 & -9.0 \\
\hline & & & & & PTGS2 & -9.1 \\
\hline & & & & & AR & -6.2 \\
\hline & & & & & CALM1 & -6.5 \\
\hline \multirow{6}{*}{ MOL000173 } & \multirow{6}{*}{ Wogonin } & \multirow{6}{*}{$\mathrm{C}_{16} \mathrm{H}_{12} \mathrm{O}_{5}$} & \multirow{6}{*}{284.28} & \multirow{6}{*}{$\begin{array}{c}632-85-9 \\
10-29-7\end{array}$} & HSP90AA1 & -6.9 \\
\hline & & & & & ESR1 & -8.5 \\
\hline & & & & & PTGS2 & -9.0 \\
\hline & & & & & AR & -6.8 \\
\hline & & & & & CALM1 & -6.7 \\
\hline & & & & & PTGS1 & -8.3 \\
\hline \multirow{5}{*}{ MOL002714 } & \multirow{5}{*}{ Baicalein } & \multirow{5}{*}{$\mathrm{C}_{15} \mathrm{H}_{10} \mathrm{O}_{5}$} & \multirow{5}{*}{270.25} & \multirow{5}{*}{$491-67-8$} & HSP90AA1 & -7.1 \\
\hline & & & & & PTGS2 & -9.5 \\
\hline & & & & & AR & -7.2 \\
\hline & & & & & CALM1 & -8.5 \\
\hline & & & & & PTGS1 & -8.8 \\
\hline \multirow{3}{*}{ MOL002773 } & \multirow{3}{*}{$\beta$-carotene } & \multirow{3}{*}{$\mathrm{C}_{40} \mathrm{H}_{56}$} & \multirow{3}{*}{536.96} & $7235-40-7$ & & \\
\hline & & & & $116-32-5$ & PTGS2 & -11.0 \\
\hline & & & & $31797-85-0$ & & \\
\hline
\end{tabular}




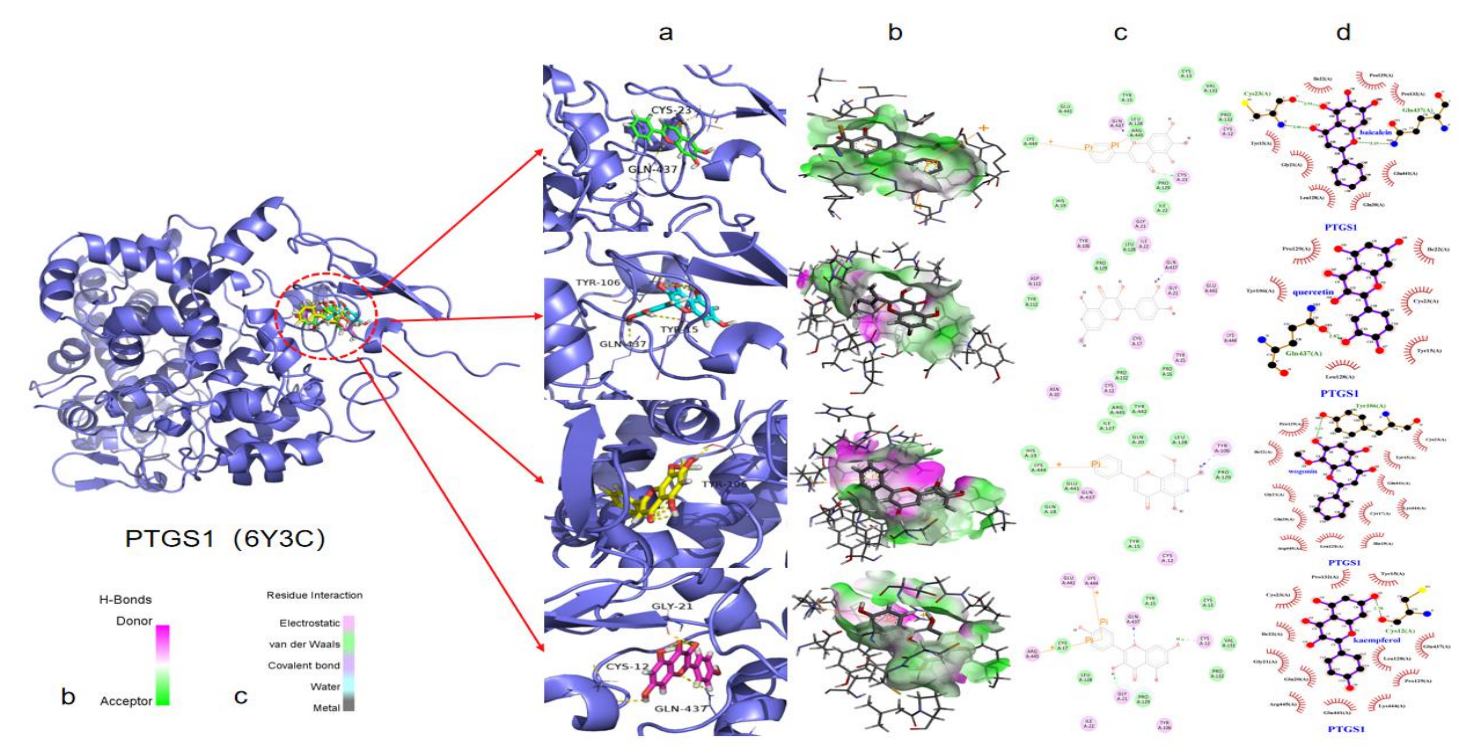

Fig 12. The compound molecule interacts with the target protein (PTGS1)

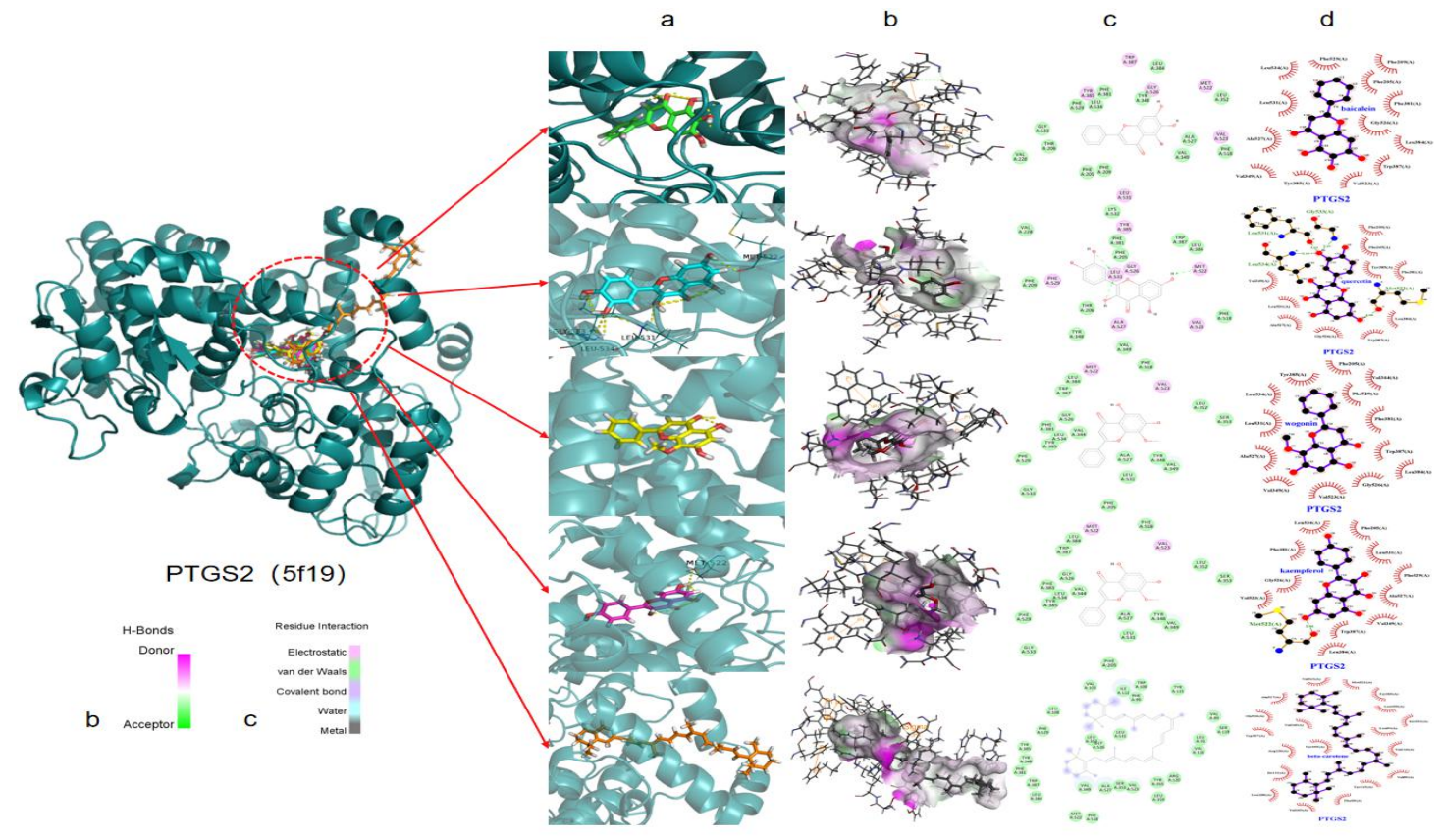

Fig 13. The compound molecule interacts with the target protein (PTGS2)

Arachidonic acid metabolism
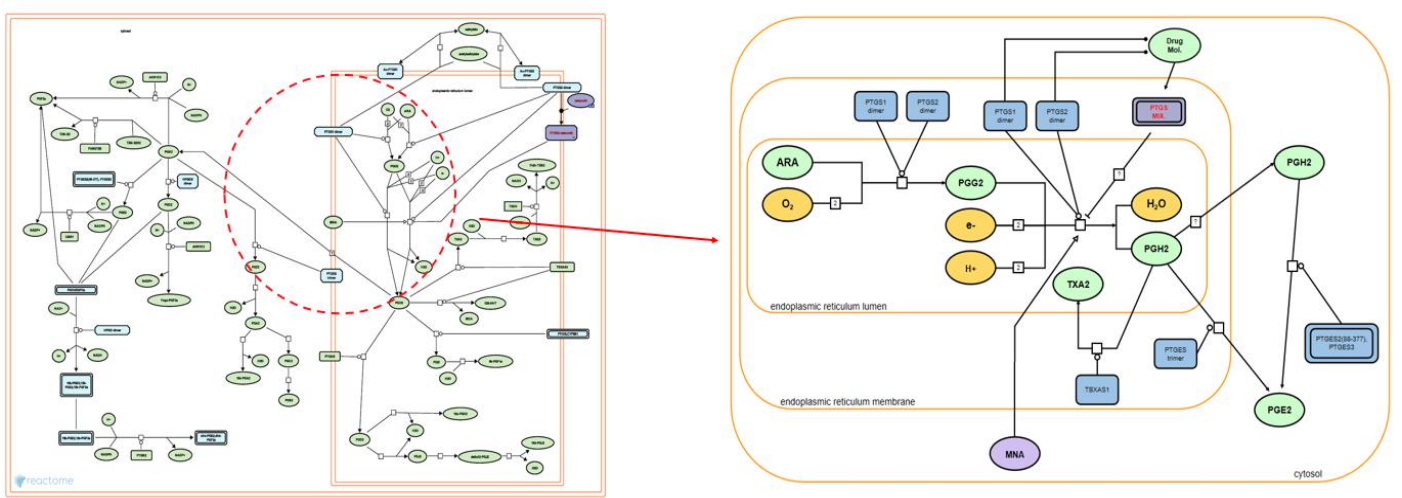

Fig 14. Arachidonic acid metabolism (R-HSA-2162123.2) 
Molecular docking diagram indicates that baicalin forms a hydrogen bond with cysteine at position 23 and glutamine at position 437 of PTGS1, and has a Pi-Pi structure and is hydrophobic to surrounding residues, but only has a hydrophobic effect with PTGS2, kaempferol forms a hydrogen bond with cysteine at position 12, glutamine at position 437, and glycine at position 21 of PTGS1 and forms a hydrogen bond with methionine at position 522 of PTGS2 and makes the surrounding residues hydrophobic, wogonin forms a hydrogen bond with tyrosine 106 of PTGS1 and makes the surrounding residues hydrophobic and Pi-Pi structure, which only has a hydrophobic effect with PTGS2, quercetin forms hydrogen bonds with PTGS1 tyrosine 15/106 and glutamine 437, and fhydrogen bonds with glycine 533, 531/534 leucine, and methionine 522 of PTGS2 making hydrophobic of surrounding residues, $\beta$-carotene only forms a covalent bond with PTGS2 and is hydrophobic.

3 Discussion

The theory of Traditional Chinese Medicine is a discipline based on system medicine. It is difficult to explain the mechanism of action of Chinese medicine by the study of a single component and target. By using bio-information data mining, Network Pharmacology, Molecular Docking to explain preliminary exploration of Chinese medicine and disease and possible molecular mechanisms, with overall innovation significance. In this study, data mining technology is used to search for common prescriptions for the treatment of osteoarthritis in clinic. The results show that "self-made prescriptions" were the main ones, so the drugs contained in the prescriptions are extracted for frequency analysis, complex network analysis, and association rules analyze and screen out core drugs and pair them, and select the best combination "Achyranthis Bidentatae Radix-Eucommiae Cortex". At present, the main compounds isolated from Achyranthis Bidentatae Radix are flavonoids, saponins, sterones, carbohydrates, etc.[6]. The main compounds in "Eucommiae Cortex" are flavonoids, lignans, phenylpropanoids, iridoids, polysaccharides, etc. Flavonoids are the intersection of two Traditional Chinese Medicines and one of the main components of the medicine. This study found 156 predictive targets and 35 active drug compounds, suggesting that the drug components have a synergistic effect and play an important role in the course of osteoarthritis, 4 drug compounds and 8 targets were selected and Estrogen Receptor (ESR1), Prostaglandin Endoperoxidase Synthase 2 (PTGS2), Androgen Receptor (AR), Heat Shock Protein (HSP90AA1), Calmodulin (CALM1), and Prostaglandin Endoperoxidase Synthase 1 (PTGS1) are both the target of drug active ingredients and the disease target of osteoarthritis according to the Degree $(\geq 10)$ and topology results. GO annotation result shows, The biological process (BP) of the positive regulation of protein kinase B may be related to the effect of drugs on osteoarthritis. Protein kinase B is a serine/threonine protein kinase that plays a key role in the P13k-Akt pathway. It has the functions of promoting cell growth, proliferation, inhibiting cell apoptosis, and promoting angiogenesis. The molecular function (MF) level shows that drug molecules can play a role through calcium ions, adenosine triphosphate enzyme, cytokines, etc. Cell level (CC) shows that this mechanism is closely related to molecular chaperone complexes, melanosomes, lysosomes, clathrin, etc. suggesting that the mechanism of drug action may be related to autophagy, Combining the results of $\mathrm{BP}, \mathrm{CC}$, and $\mathrm{MF}$, it can be concluded that the drug molecule directly or indirectly exerts the biological behavior of anti-osteoarthritis by binding to different targets.

KEGG pathway analysis of key nodes further showed that drug molecules can pass through the Estrogen signaling pathway (ESR1), Arachidonic acid metabolism (PTGS1/PTGS2), Renin secretion (AR), and IL-17 signaling pathway (IL-17) and other pathways have regulatory effects, Arachidonic acid metabolism pathway has been studied in recent years and occupies a dominant position, Fig 15, Arachidonic acid (ARA) undergoes a redox reaction under the action of PTGS1 and PTGS2 in the 
endoplasmic reticulum cavity to generate prostaglandin $\mathrm{H} 2$ (PGH2) and diffuse it to the active site of the endoplasmic reticulum membrane prostaglandin E synthase (PTGES). With the aid of glutathione (GSH), PGH2 is formed into prostaglandin E2 (PGE2)[7,8]. A part of PGH2 diffuses directly into the cytoplasm through the endoplasmic reticulum membrane and is heterogeneous to form PGE2 by PTGES2 and PTGES3, while a part of PGH2 in the endoplasmic reticulum is heterogeneous to form thromboxane A2 (TXA2) by TBXAS1[9,10], Peng[11]detected that the levels of IL-18 and PGE2 in the joint fluid of patients with osteoarthritis were significantly higher than those of normal people, Hossein[12], Jun[13]reduce the synthesis of PGE2 and TXA2 by inhibiting and down-regulating the expression of PTGS2 gene to slow down the process of osteoarthritis. Estrogen diffuses freely into the cell and binds to the estrogen receptor $(\mathrm{ER} \alpha / \beta)$ in the nucleus to regulate the proliferation and inhibition of downstream genes in the classic mechanism of estrogen [14]. In bone tissue, the content of ER $\beta$ in nuclear receptors is higher than that of ER $\alpha$. ER $\alpha$ regulates the growth of osteoblasts, while ER $\beta$ is involved in bone formation and resorption. In the absence of ligands, ER contains HSP90 and P23. The complex with chaperone-related proteins releases ERs and HSP90 under the action of ATP hydrolase[15], HSP40 recaptures ERs through ligand binding and recaptures the chaperone complex recombination. The ligand can compete to bind to the HSP40 site to escape chaperone recombination and DNA binding[15]. LIU[16] found through in vitro experiments that the binding of estradiol to ER $\alpha$ inhibits the activation of ERK signaling pathway, promotes chondrocyte autophagy, inhibits apoptosis, and regulates chondrocyte proliferation. The classic mechanism of Renin secretion is involved in the regulation of blood pressure. With the discovery of RAAS components in bone tissue, the function of stimulating the formation of osteoclasts and inhibiting the activity of osteoblasts gradually appears[17]. The level of renin is positively correlated with the degree of osteoarthritis in the synovial tissue of osteoarthritis[18,19], involving in the formation of inflammation and the hypertrophy of chondrocytes[20]. Wang[21] found the up-regulation of angiotensinogen (AGT) in cartilage tissue of osteoarthritis was detected by protein electrophoresis. Knockout of AGT cannot activate the JAK2/STAT3 pathway, inhibit the increase of IL-6 and MMP-13 in chondrocytes, and regulate bone Inflammatory response of arthritis, Interleukin-17 contains 6 subtypes (ILA-F) which are cytokines secreted by a variety of cells[22](T cells, NKT cells, mast cells, neutrophils, etc.). It is also an inflammation-initiating factor induced by $\mathrm{T}$ cells. After binding to membrane receptors, it stimulates $\mathrm{T}$ cells to secrete interleukins (IL-1, 4, 5, 6, 13) and tumors through MAP kinase and nuclear transcription factor (NF-kB) pathways. Tumor necrosis factor (TNF- $\alpha$ ), matrix metalloproteinases (MMP1, 3, 9, 13), chemokines (CXCL1,2,5; CCL2, 5), etc. Several studies have shown that[23,24] IL-17 levels are related to bone loss around joints and local bone defects. The RANKL pathway induces osteoclast formation[25,26], which plays a central role in articular cartilage damage.

Among the five core drug molecules predicted in this study, the current research is as follows: quercetin[27,28] and kaempferol[29] can inhibit the increase of Matrix Metalloproteinases (MMPs) and PGE2 in joints induced by interleukin, HSP90 can interfere with vascular endothelial growth factor Flk-1 receptor and affect the angiogenesis of bone growth plate, quercetin[30] prevent the up-regulation of HSP70, but does not reduce the level of HSP90, wogonin[31] and baicalin[31,32] inhibit the production pathway of PGE2 induced by lipopolysaccharide, reduce the synthesis of PGE2, wogonin[28,33] has been proven to reduce the synthesis of PGE2 by activating the ROS/ERK/Nrf2 pathway of chondrocytes to exert anti-inflammatory and chondroprotective functions, $\beta$-carotene[34] inhibits lipopolysaccharide from stimulating inflammatory genes in macrophages by inhibiting the activation of redox group NF-kB, for the interaction mechanism between the drug and the target 
predicted in this study, further experiments are needed to verify.

The complexity of the components of Traditional Chinese Medicine has always been a limitation of the scientific research of Traditional Chinese Medicine. There is still a lack of effective relevant research reports on the treatment of osteoarthritis with Traditional Chinese Medicine. This study uses data mining, network pharmacology, and molecular docking technology to predict the key mechanism of disease target interaction, Showing the overall characteristics of "Achyranthis Bidentatae Radix-Eucommiae Cortex" in the treatment of osteoarthritis. The mechanism of action and safety evaluation of Network Pharmacology have a wide range of application value, and provide a theoretical basis and direction for the subsequent screening of pharmaceutical ingredients to establish a quantitative fingerprint to improve its quality evaluation.

4 Conclusion

"Eucommia bark - achyranthes" drug molecules can be combined with the target to produce hydrogen bond, hydrophobic function and Pi-Pi directly or indirectly affecting the corresponding targets, to participate in the regulation of osteogenesis and osteoclast proliferation, protect the extracellular matrix, inhibition of cell apoptosis and anti-inflammatory for resistance to osteoarthritis, also, providing the basis for interpretation of its action mechanism.

\section{Abbreviations}

ECM: Extracellular Matrix

ACAN: Aggrecan

OB: Oral Absorption bioavailability

DL: Drug-like

GO: Gene Ontology

PPI: Protein-protein Interaction

KEGG: Kyoto Encyclopedia of Genes and Genomes

BP: Biological Process

CC: Cellular Component

MF: Molecular Function

PTGS 1/2: Prostaglandin Endoperoxidase 1/2

HSP90: Heat Shock Protein 90

CALM 1: Calmodulin 1

AR: Androgen Receptor

ESR 1: Estrogen Receptor

ARA: Arachidonic Acid

GSH: Glutathione

TXA2: Thromboxane A2

RAAS: Renin-angiotensin-aldosterone System

TNF: Tumor Necrosis Factor

MMPs: Matrix Metalloproteinases

IL: Interleukin

AGT: Angiotensinogen 


\section{* Declarations}

- Ethics approval and consent to participate

Not applicable.

- Consent for publication

Not applicable.

- Availability of data and materials

Ancient and Modern Medical Record Cloud Platform: http://www.yiankb.com/ ;

Traditional Chinese Medicine Systems Pharmacology Database and Analysis Platform (TCMSP):

http://tcmspw.com/tcmsp.php;

Genecard: https://www.genecards.org/;

OMIM: https://omim.org/;

STRING: https://string-db.org/ ;

JAVA version 1.8.0_171

Gephi version 0.9.2

Python version 2.7.6

PyMol verson 2.4.0

AutoDockTools version 1.5.6

AutoDock Vina version 1.1.2

Cytoscape version 3.6.0

R Project version 4.0.2

DiscoveryStudio version 3.5

LigPlus version 2.2

PyCharm Community Edition Version 2020.1.2

Strawberry-Perl version 5.32.0.

- Competing interests

All authors certify that they have no affiliations with or involvement in any organization or entity with any financial interest or non-financial interest in the subject matter or materials discussed in this manuscript.

- Funding

The authors did not receive support from any organization for the submitted work.

- Authors' contributions

YANG J. mainly responsible for data processing, chart production, article writing. (JYang9527@hotmail.com)

SUN Y. CHEN C. proofread the data, graphs, and tables in the article again, and review the professional knowledge in the article.

Other authors: According to the inclusion and exclusion criteria, data collection, technical support, collation and article proofreading were carried out.

- Acknowledgements

First of all, I sincerely thank OMIN, Genecard, STRING, and AMMRCP for the open resource acquisition; secondly, thank the authors of the article for their joint efforts; finally, thank Qu Chao for her technical support.

- Authors' information (optional)

Shenyang Orthopedics Hospital, NO.115, Dongbei Road, Dadong District, Shenyang City, Liaoning Province, China 
Liaoning Traditional Chinese Medicine University, NO.79 Chongshan Road,Shenyang City Liaoning Province, China

Corresponding authors: Correspondence to SUN Yu ( 357602739@qq.com; sunyu0629@sina.com )

\section{References:}

1. Abramoff B, Caldera FE. (2020). Osteoarthritis: Pathology, Diagnosis, and Treatment Options. Med Clin North Am, 104(2), 293-311. doi: 10.1016/j.mcna.2019.10.007

2. Boehme KA, Rolauffs B. (2018). Onset and Progression of Human Osteoarthritis-Can Growth Factors, Inflammatory Cytokines, or Differential miRNA Expression Concomitantly Induce Proliferation, ECM Degradation, and Inflammation in Articular Cartilage? INT J MOL SCI, 19(8). doi: 10.3390/ijms19082282

3. Sanchez-Adams J, Leddy HA, McNulty AL, O'Conor CJ, Guilak F. (2014). The mechanobiology of articular cartilage: bearing the burden of osteoarthritis. CURR RHEUMATOL REP, 16(10), 451. doi: 10.1007/s11926-014-0451-6

4. Loeser RF, Collins JA, Diekman BO. (2016). Ageing and the pathogenesis of osteoarthritis. NAT REV RHEUMATOL, 12(7), 412-420. doi: 10.1038/nrrheum.2016.65

5. Murphy C, Withrow J, Hunter M, Liu Y, Tang YL, Fulzele S, Hamrick MW. (2018). Emerging role of extracellular vesicles in musculoskeletal diseases. MOL ASPECTS MED, 60, 123-128. doi: 10.1016/j.mam.2017.09.006

6. Wang J, Ran Q, Zeng HR, Wang L, Hu CJ, Huang QW. (2018). Cellular stress response mechanisms of Rhizoma coptidis: a systematic review. Chin Med, 13, 27. doi: 10.1186/s13020-018-0184-y

7. Dong L, Vecchio AJ, Sharma NP, Jurban BJ, Malkowski MG, Smith WL. (2011). Human cyclooxygenase-2 is a sequence homodimer that functions as a conformational heterodimer. J BIOL CHEM, 286(21), 19035-19046. doi: 10.1074/jbc.M111.231969

8. Yoshikawa K, Takei S, Hasegawa-Ishii S, Chiba Y, Furukawa A, Kawamura N, Hosokawa M, Woodward DF, Watanabe K, Shimada A. (2011). Preferential localization of prostamide/prostaglandin F synthase in myelin sheaths of the central nervous system. BRAIN RES, 1367, 22-32. doi: 10.1016/j.brainres.2010.10.019

9. Chevalier D, Lo-Guidice JM, Sergent E, Allorge D, Debuysere H, Ferrari N, Libersa C, Lhermitte M, Broly F. (2001). Identification of genetic variants in the human thromboxane synthase gene (CYP5A1). Mutat Res, 432(3-4), 61-67. doi: 10.1016/s1383-5726(00)00004-2

10. Miyata A, Yokoyama C, Ihara H, Bandoh S, Takeda O, Takahashi E, Tanabe T. (1994). Characterization of the human gene (TBXAS1) encoding thromboxane synthase. Eur J Biochem, 224(2), 273-279. doi: 10.1111/j.1432-1033.1994.00273.x

11. Peng CZ, Cao JM, Xiao T, Peng C, Yang HB, Chen X, Fang JZ. (2006). [Concentration of IL-18 and PGE2 in synovial fluid in patients with osteoarthritis and its significance]. Zhong Nan Da Xue Xue Bao Yi Xue Ban, 31(6), 862-865

12. Maghsoudi H, Hallajzadeh J, Rezaeipour M. (2018). Evaluation of the effect of polyphenol of escin compared with ibuprofen and dexamethasone in synoviocyte model for osteoarthritis: an in vitro study. CLIN RHEUMATOL, 37(9), 2471-2478. doi: 10.1007/s10067-018-4097-z

13. Aikawa J, Uchida K, Takano S, Inoue G, Iwase D, Miyagi M, Mukai M, Shoji S, Sekiguchi H, Takaso M. (2018). Regulation of calcitonin gene-related peptide expression through the COX-2/mPGES-1/PGE2 pathway in the infrapatellar fat pad in knee osteoarthritis. LIPIDS 
HEALTH DIS, 17(1), 215. doi: 10.1186/s12944-018-0864-8

14. Hah N, Kraus WL. (2014). Hormone-regulated transcriptomes: lessons learned from estrogen signaling pathways in breast cancer cells. MOL CELL ENDOCRINOL, 382(1), 652-664. doi: 10.1016/j.mce.2013.06.021

15. Smith DF, Toft DO. (2008). Minireview: the intersection of steroid receptors with molecular chaperones: observations and questions. MOL ENDOCRINOL, 22(10), 2229-2240. doi: 10.1210/me.2008-0089

16. Liu M, Xie W, Zheng W, Yin D, Luo R, Guo F. (2019). [Targeted binding of estradiol with ESR1 promotes proliferation of human chondrocytes in vitro by inhibiting activation of ERK signaling pathway]. Nan Fang Yi Ke Da Xue Xue Bao, 39(2), 134-143. doi: 10.12122/j.issn.1673-4254.2019.09.02

17. Mo C, Ke J, Zhao D, Zhang B. (2020). Role of the renin-angiotensin-aldosterone system in bone metabolism. J BONE MINER METAB. doi: 10.1007/s00774-020-01132-y

18. Wu Y, Zeng Y, Li M, Liu Y, Yang J, Shen B. (2020). [Expressions of Renin, angiotensin converting enzyme, angiotensin receptor 1 , and angiotensin receptor 2 in synovial tissue of osteoarthritis at different stages]. Zhongguo Xiu Fu Chong Jian Wai Ke Za Zhi, 34(3), 362-366. doi: $10.7507 / 1002-1892.201904065$

19. Wu Y, Li M, Zeng J, Feng Z, Yang J, Shen B, Zeng Y. (2020). Differential Expression of Renin-Angiotensin System-related Components in Patients with Rheumatoid Arthritis and Osteoarthritis. AM J MED SCI, 359(1), 17-26. doi: 10.1016/j.amjms.2019.10.014

20. Wu Y, Lu X, Li M, Zeng J, Zeng J, Shen B, Zeng Y. (2019). Renin-angiotensin system in osteoarthritis: A new potential therapy. INT IMMUNOPHARMACOL, 75, 105796. doi: 10.1016/j.intimp.2019.105796

21. Wang W, Han X, Zhao T, Zhang X, Qu P, Zhao H. (2020). AGT, targeted by miR-149-5p, promotes IL-6-induced inflammatory responses of chondrocytes in osteoarthritis via activating JAK2/STAT3 pathway. CLIN EXP RHEUMATOL

22. Miossec P, Kolls JK. (2012). Targeting IL-17 and TH17 cells in chronic inflammation. NAT REV DRUG DISCOV, 11(10), 763-776. doi: 10.1038/nrd3794

23. Rossini M, Viapiana O, Adami S, Idolazzi L, Fracassi E, Gatti D. (2016). Focal bone involvement in inflammatory arthritis: the role of IL17. RHEUMATOL INT, 36(4), 469-482. doi: 10.1007/s00296-015-3387-x

24. Kobayashi M, Squires GR, Mousa A, Tanzer M, Zukor DJ, Antoniou J, Feige U, Poole AR. (2005). Role of interleukin-1 and tumor necrosis factor alpha in matrix degradation of human osteoarthritic cartilage. Arthritis Rheum, 52(1), 128-135. doi: 10.1002/art.20776

25. Kim HJ, Seo SJ, Kim JY, Kim YG, Lee Y. (2020). IL-17 promotes osteoblast differentiation, bone regeneration, and remodeling in mice. Biochem Biophys Res Commun, 524(4), 1044-1050. doi: 10.1016/j.bbrc.2020.02.054

26. Huang Z, Pei X, Graves DT. (2020). The Interrelationship Between Diabetes, IL-17 and Bone Loss. CURR OSTEOPOROS REP, 18(1), 23-31. doi: 10.1007/s11914-020-00559-6

27. Sung MS, Lee EG, Jeon HS, Chae HJ, Park SJ, Lee YC, Yoo WH. (2012). Quercetin inhibits IL-1beta-induced proliferation and production of MMPs, COX-2, and PGE2 by rheumatoid synovial fibroblast. INFLAMMATION, 35(4), 1585-1594. doi: 10.1007/s10753-012-9473-2

28. Shen SC, Lee WR, Lin HY, Huang HC, Ko CH, Yang LL, Chen YC. (2002). In vitro and in vivo inhibitory activities of rutin, wogonin, and quercetin on lipopolysaccharide-induced nitric oxide 
and prostaglandin E(2) production. EUR J PHARMACOL, 446(1-3), 187-194. doi: 10.1016/s0014-2999(02)01792-2

29. Yoon HY, Lee EG, Lee H, Cho IJ, Choi YJ, Sung MS, Yoo HG, Yoo WH. (2013). Kaempferol inhibits IL-1beta-induced proliferation of rheumatoid arthritis synovial fibroblasts and the production of COX-2, PGE2 and MMPs. INT J MOL MED, 32(4), 971-977. doi: 10.3892/ijmm.2013.1468

30. Genin O, Hasdai A, Shinder D, Pines M. (2012). The effect of inhibition of heat-shock proteins on thiram-induced tibial dyschondroplasia. Poult Sci, 91(7), 1619-1626. doi: 10.3382/ps.2012-02207

31. Chen YC, Shen SC, Chen LG, Lee TJ, Yang LL. (2001). Wogonin, baicalin, and baicalein inhibition of inducible nitric oxide synthase and cyclooxygenase-2 gene expressions induced by nitric oxide synthase inhibitors and lipopolysaccharide. BIOCHEM PHARMACOL, 61(11), 1417-1427. doi: 10.1016/s0006-2952(01)00594-9

32. Suh MG, Choi HS, Cho K, Park SS, Kim WJ, Suh HJ, Kim H. (2020). Anti-inflammatory action of herbal medicine comprised of Scutellaria baicalensis and Chrysanthemum morifolium. Biosci Biotechnol Biochem, 84(9), 1799-1809. doi: 10.1080/09168451.2020.1769464

33. Khan NM, Haseeb A, Ansari MY, Devarapalli P, Haynie S, Haqqi TM. (2017). Wogonin, a plant derived small molecule, exerts potent anti-inflammatory and chondroprotective effects through the activation of ROS/ERK/Nrf2 signaling pathways in human Osteoarthritis chondrocytes. Free Radic Biol Med, 106, 288-301. doi: 10.1016/j.freeradbiomed.2017.02.041

34. Bai SK, Lee SJ, Na HJ, Ha KS, Han JA, Lee H, Kwon YG, Chung CK, Kim YM. (2005). beta-Carotene inhibits inflammatory gene expression in lipopolysaccharide-stimulated macrophages by suppressing redox-based NF-kappaB activation. EXP MOL MED, 37(4), 323-334. doi: $10.1038 / \mathrm{emm} .2005 .42$ 
Figures

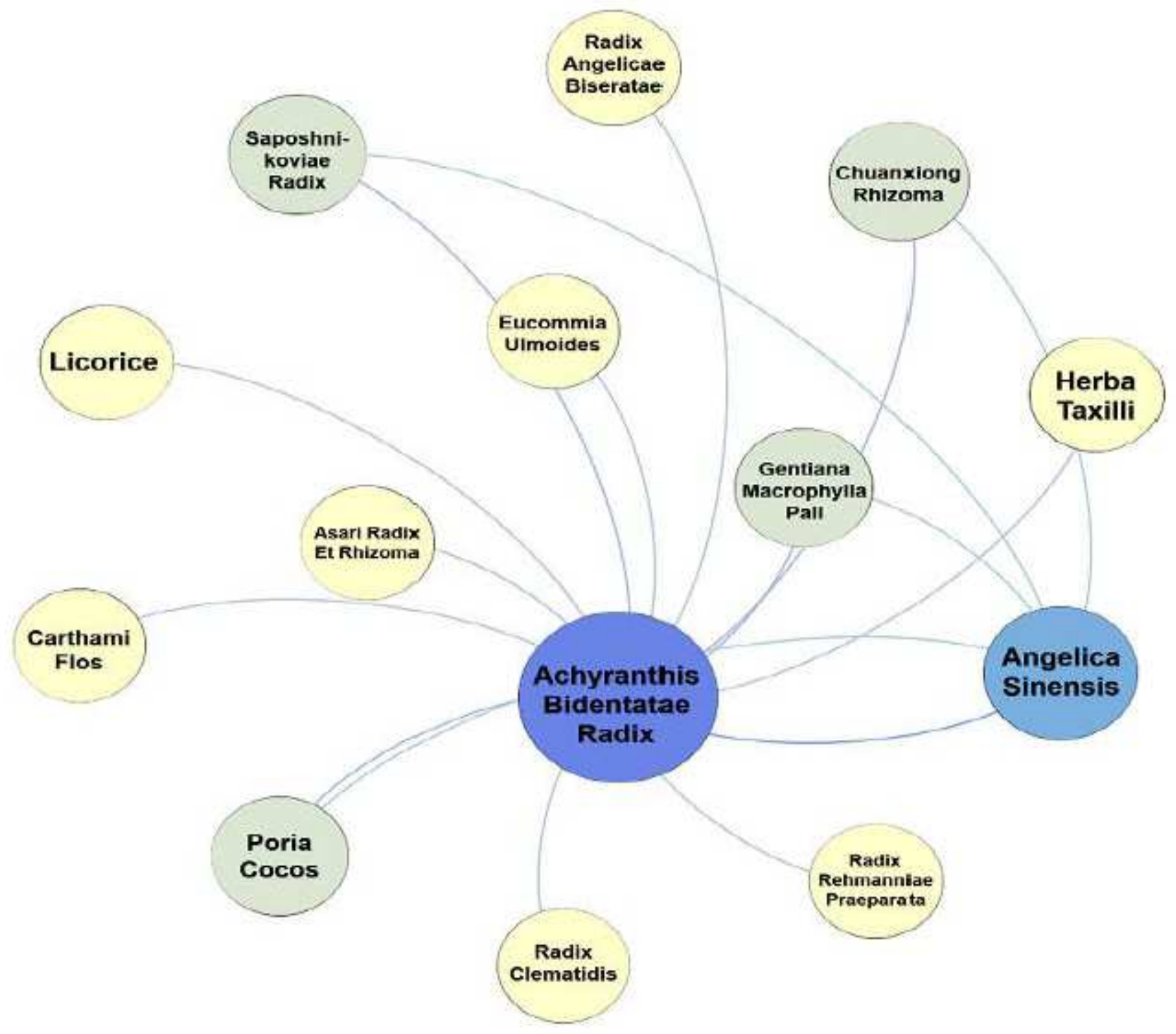

Figure 1

Association Analysis 


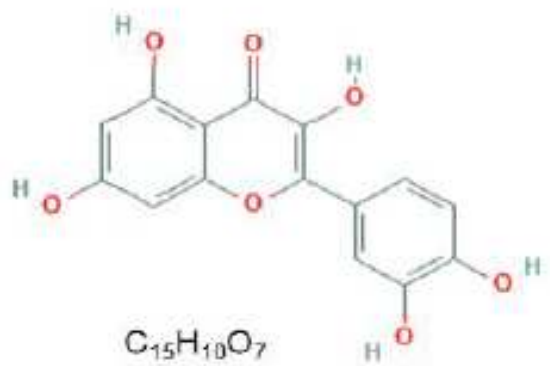

a. Quercetin

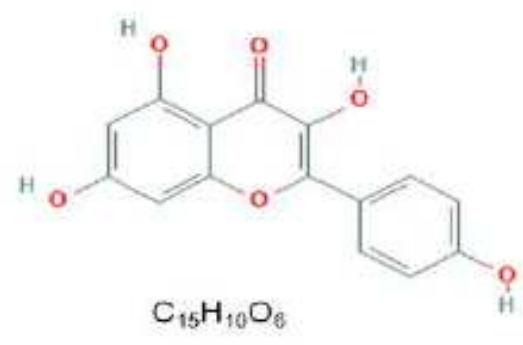

b. Kaempferol

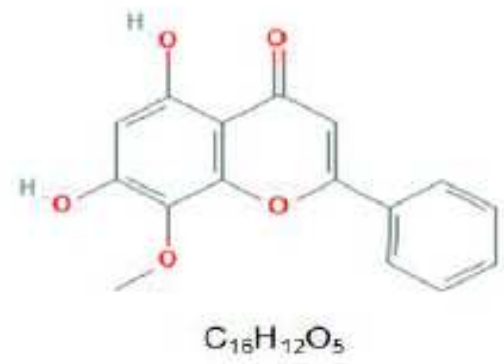

c. Wogonin<smiles>O=c1cc(-c2ccccc2)oc2cc(O)c(O)c(O)c12</smiles>

d. Baicalein

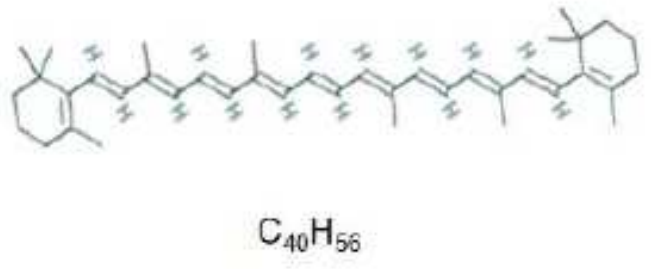

e. $\beta$-carotene

Figure 2

The top 5 compounds 


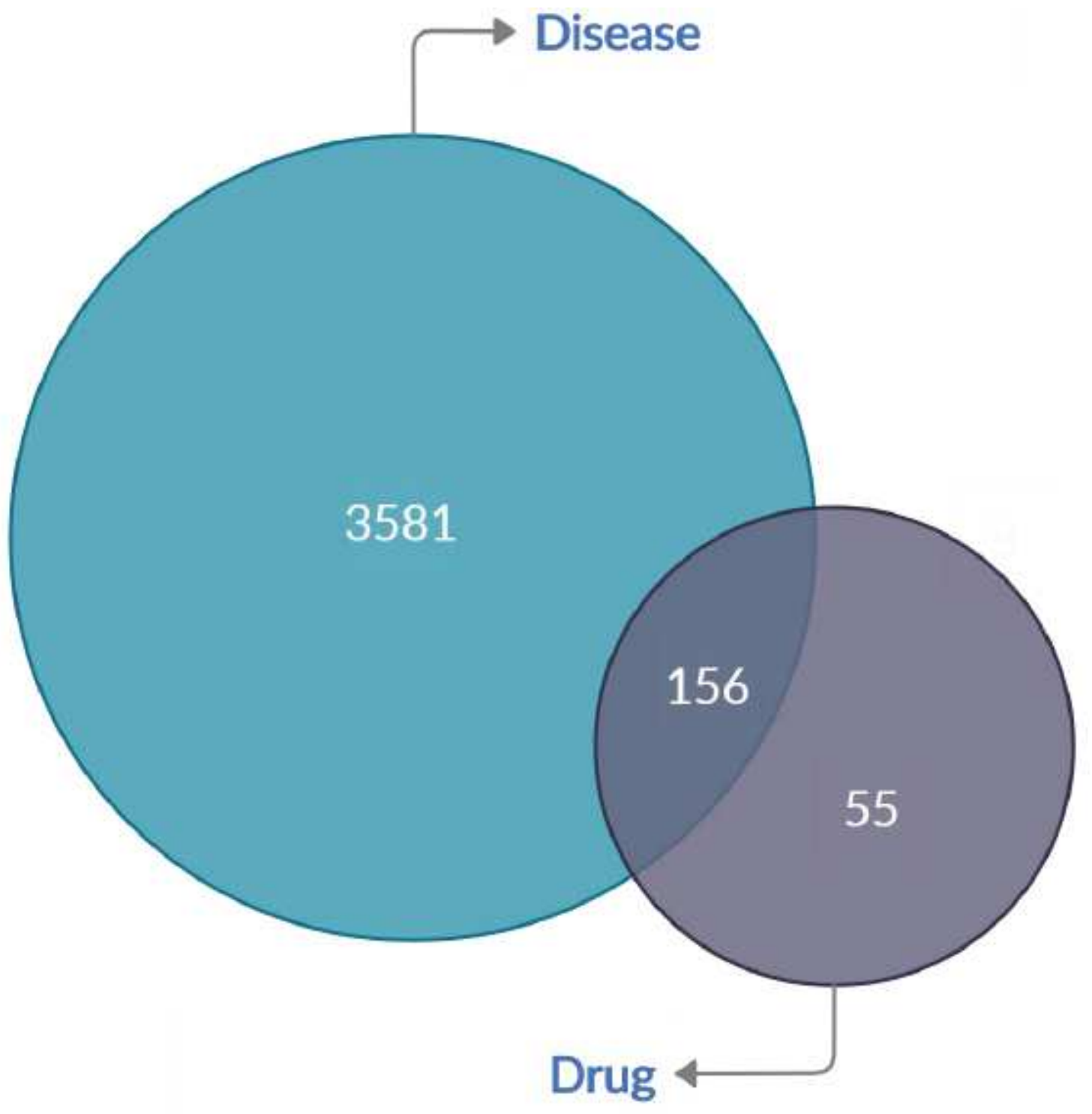

Figure 3

Venn diagram 


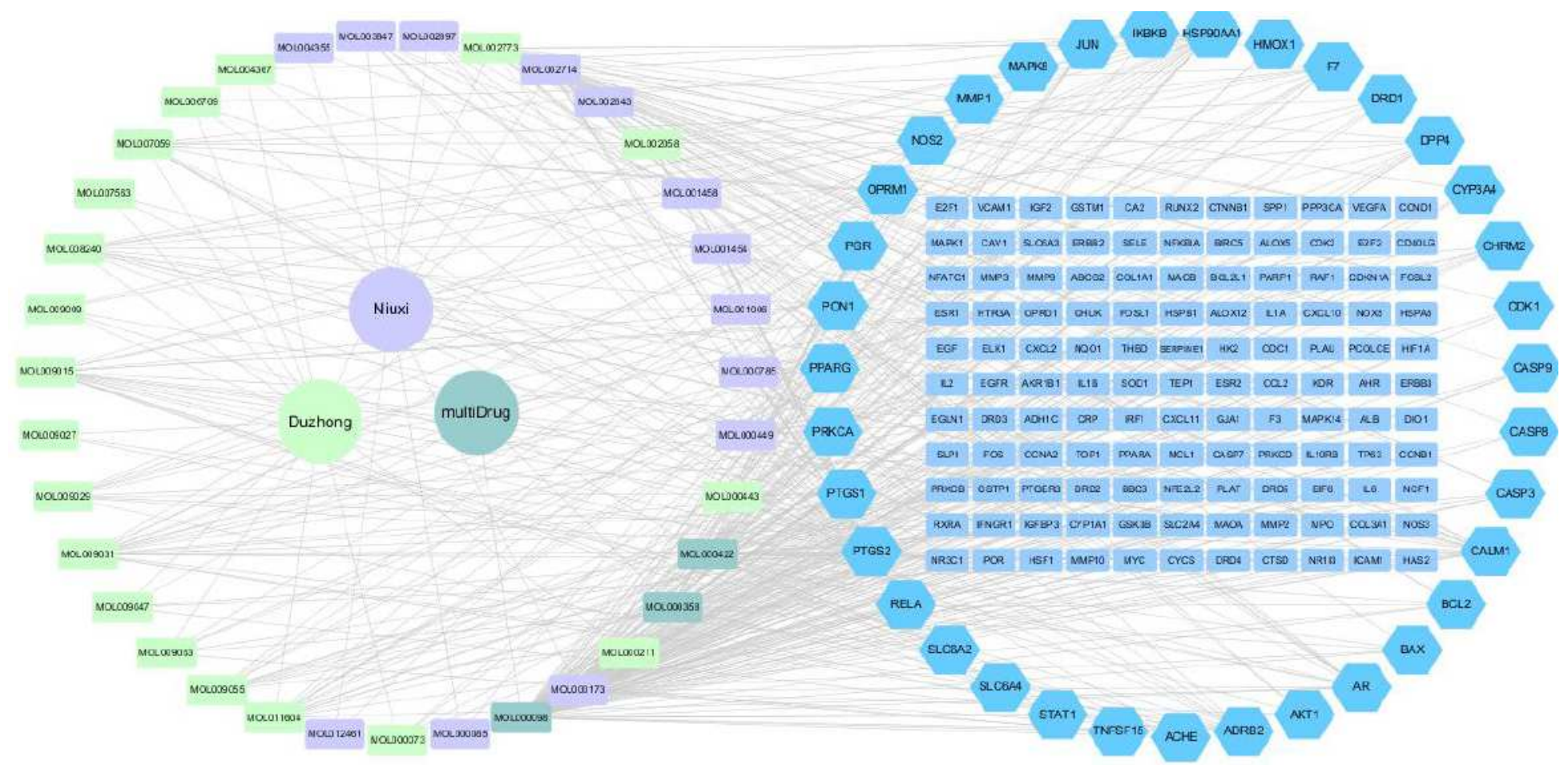

\section{Figure 4}

Compound and component target network 


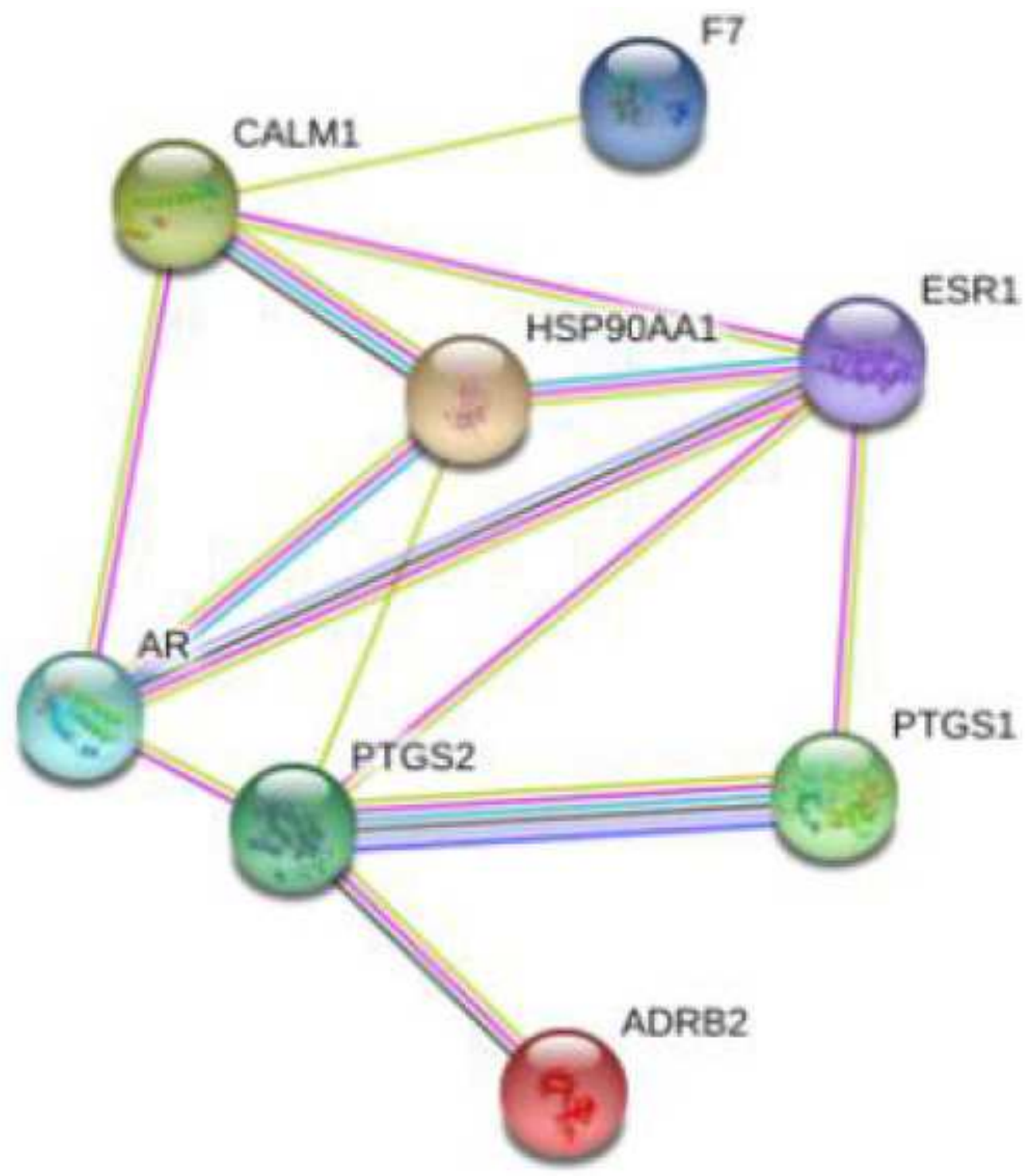

Figure 5

PPI network 


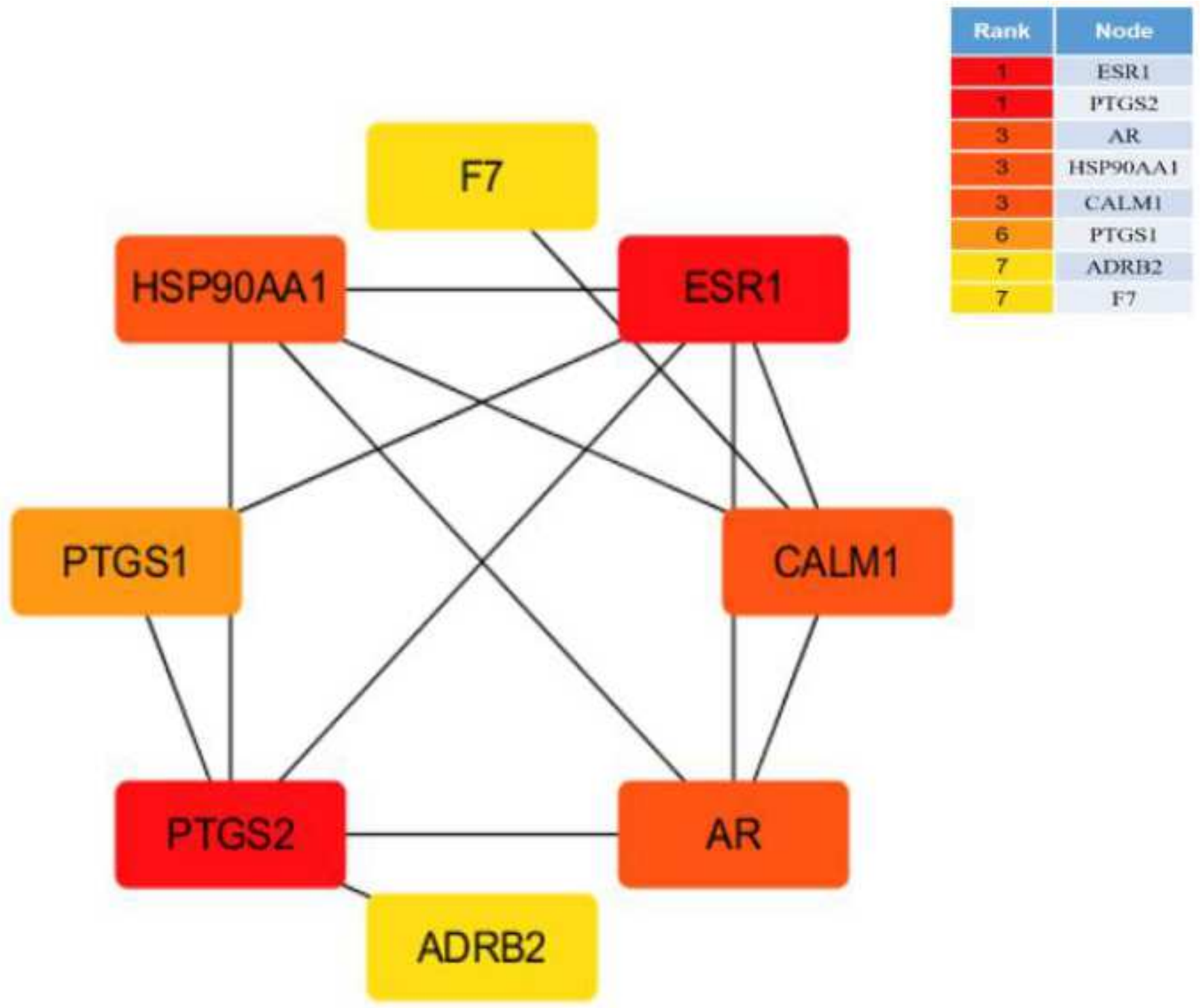

Figure 6

Cytohubba Visualization 

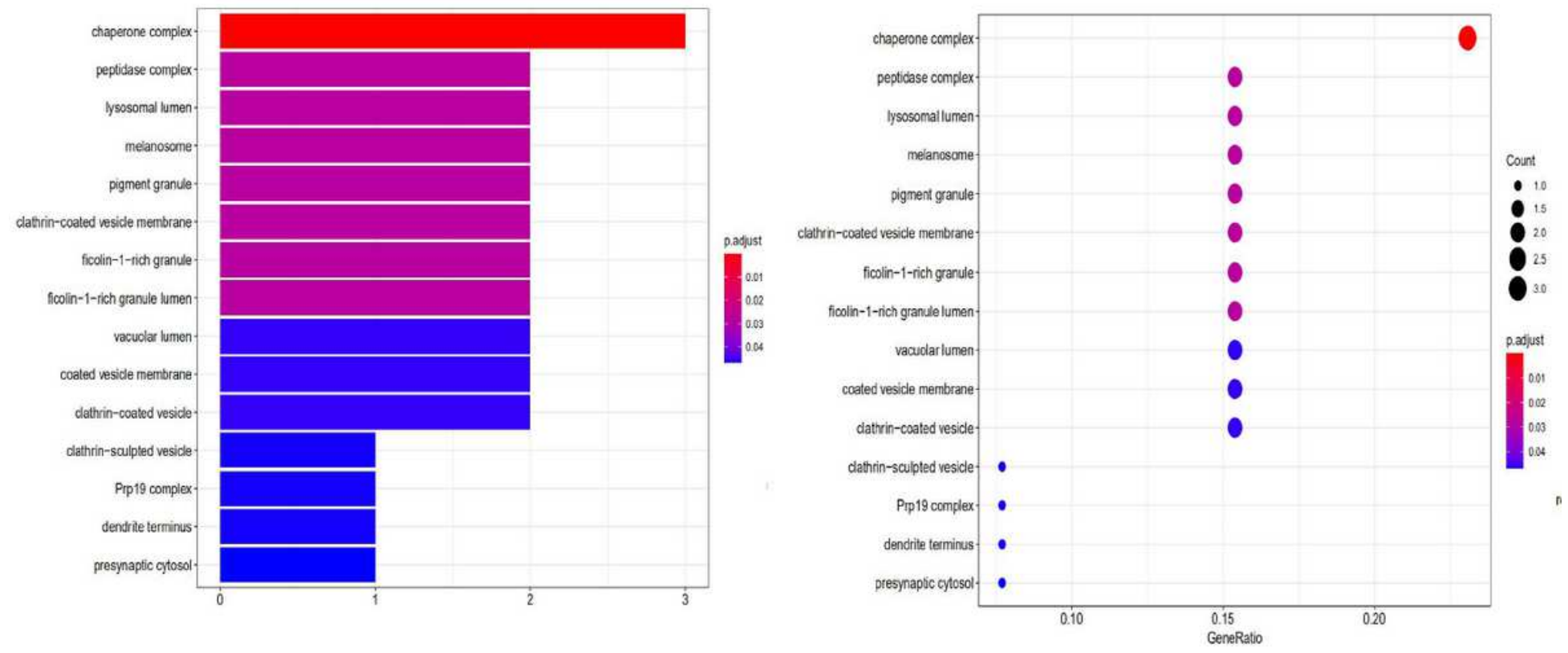

Figure 7

GO-CC Bar graph, GO-CC Bubble
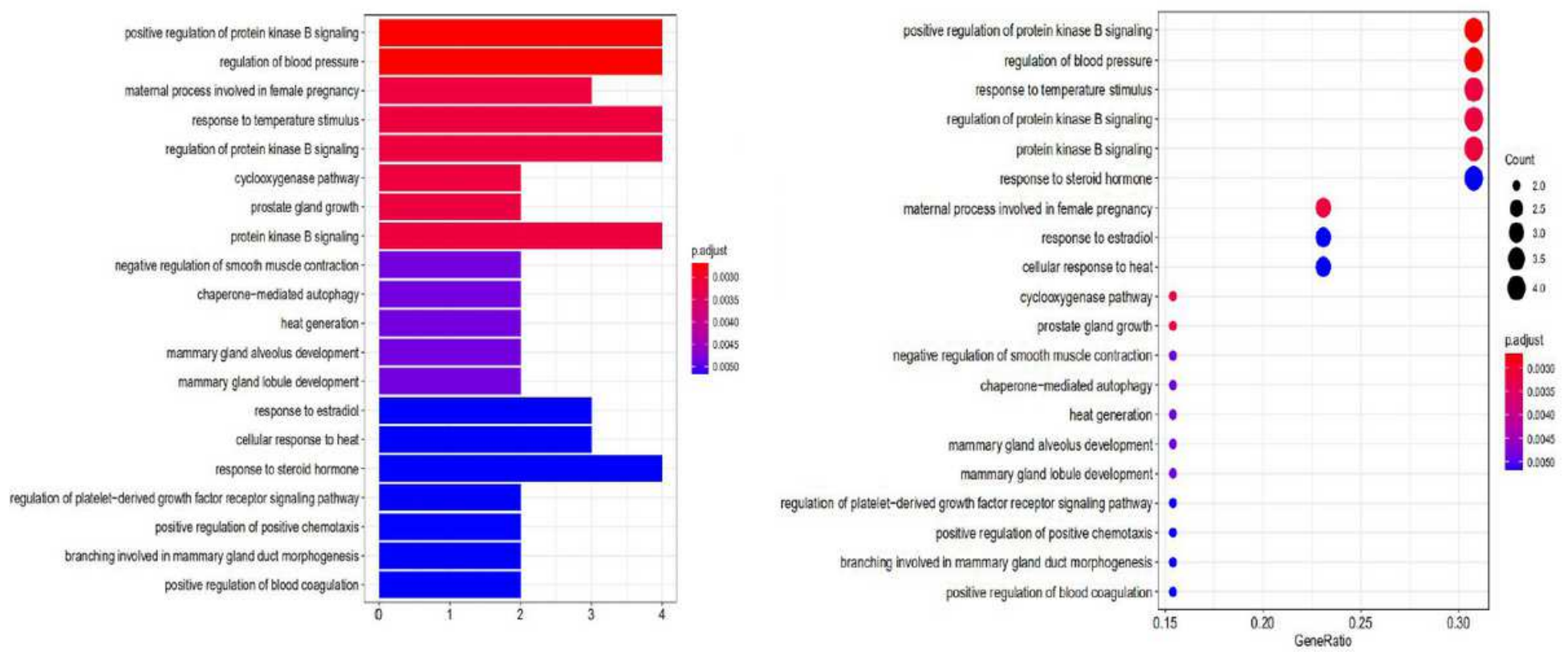

Figure 8

GO-BP Bar graph, GO-BP Bubble 

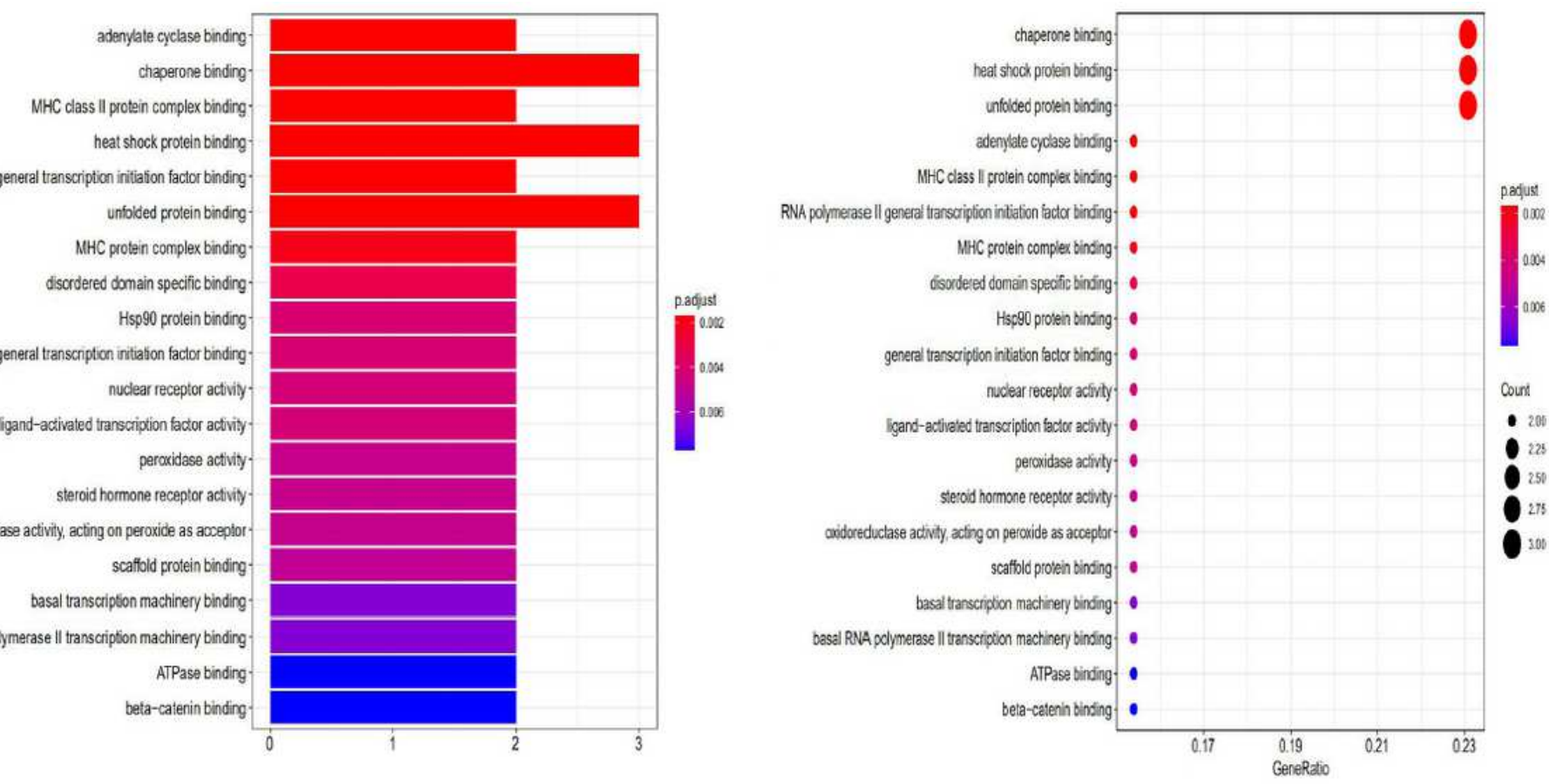

\section{Figure 9}

GO-MF Bar graph, GO-MF Bubble
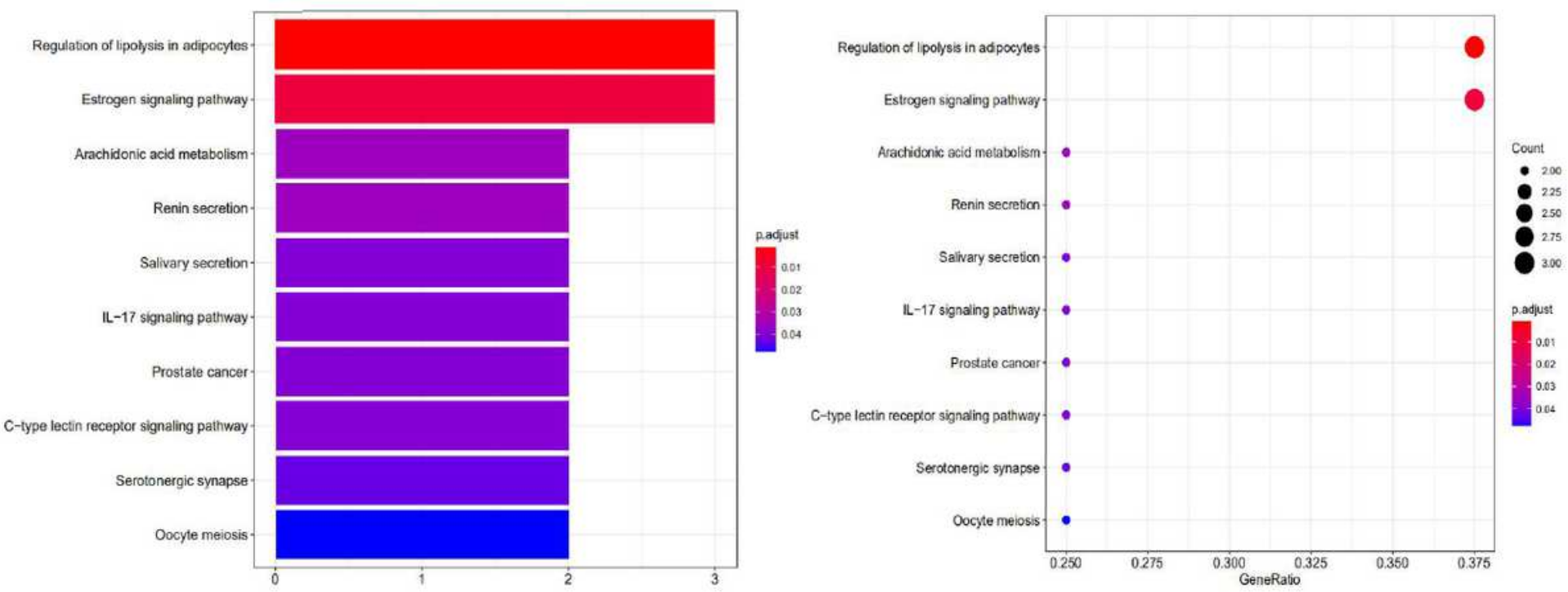

Figure 10

KEGG Bar graph, KEGG Bubble 


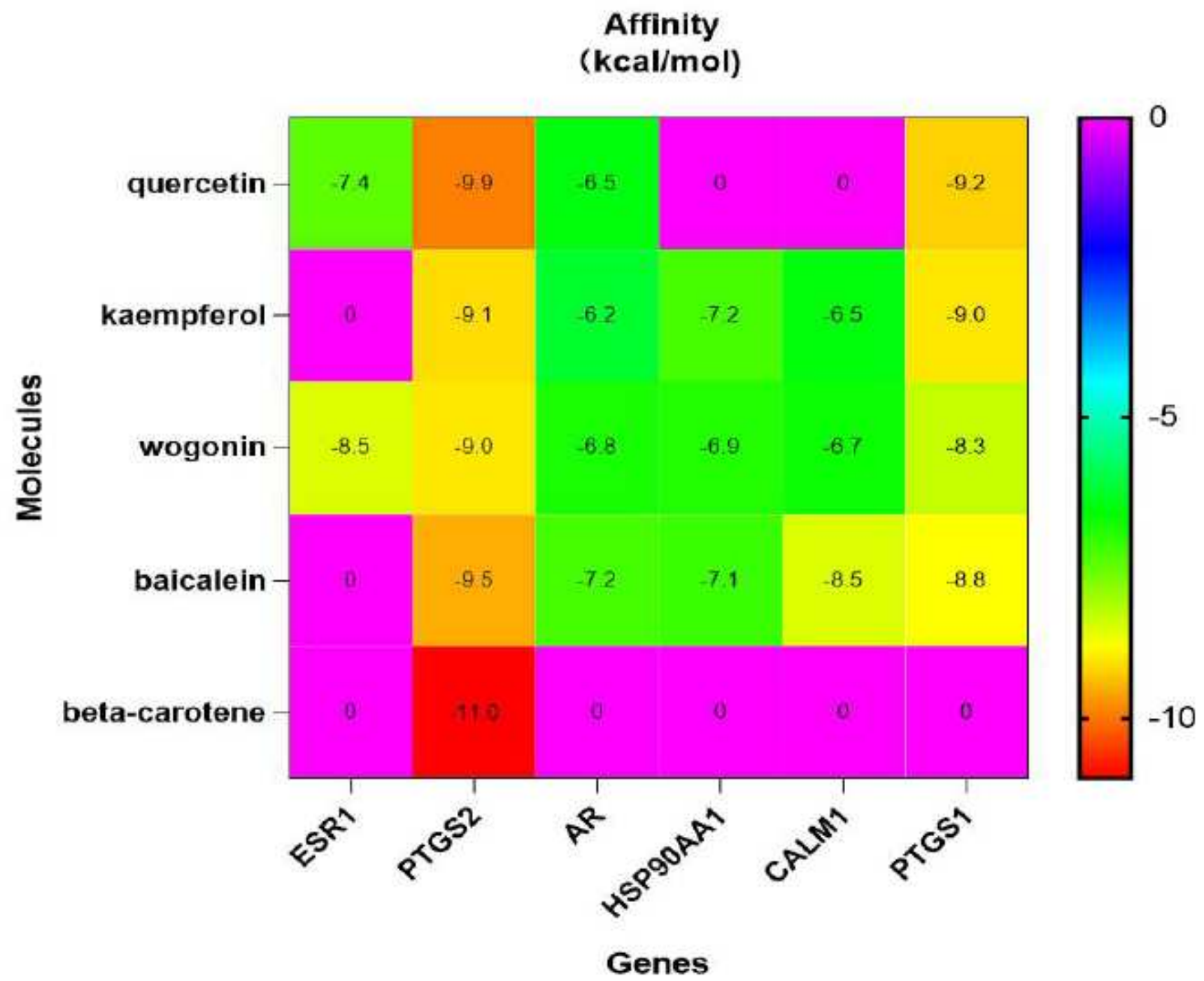

Figure 11

Affinity heat map 


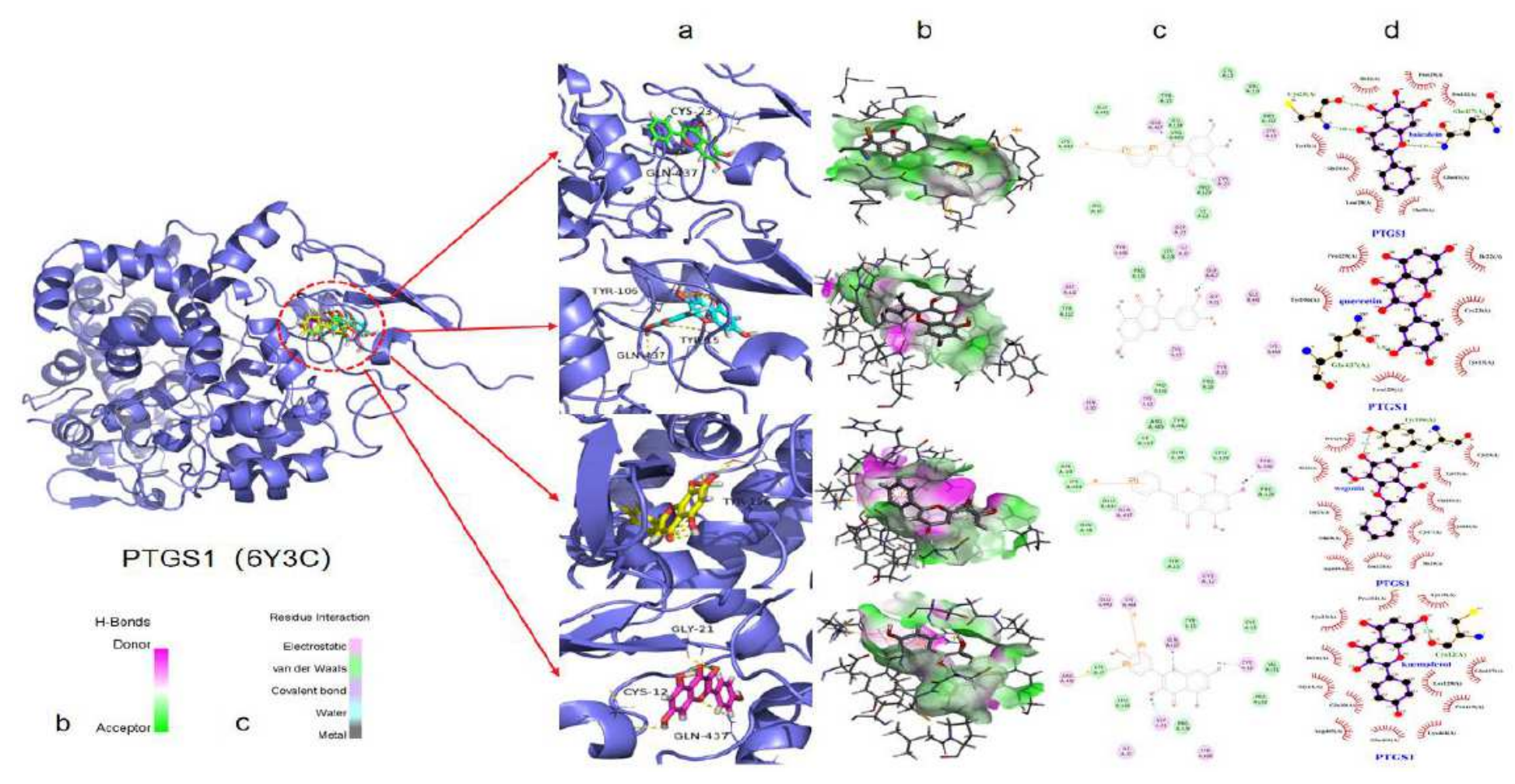

Figure 12

The compound molecule interacts with the target protein (PTGS1)

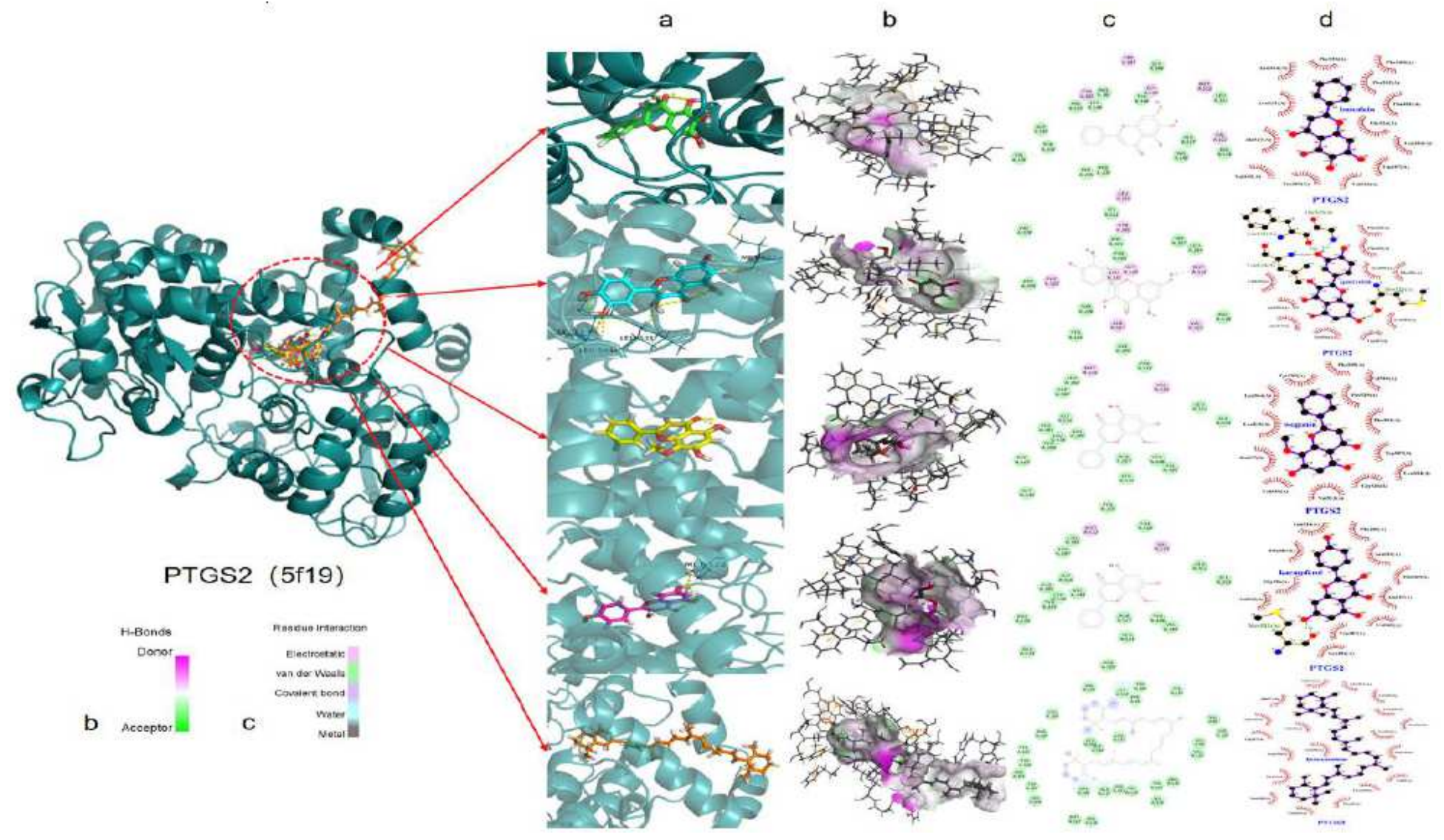

Figure 13 
The compound molecule interacts with the target protein (PTGS2)

Arachidonic acid metabolism

(hse00590,R-HSA-2162123.2)
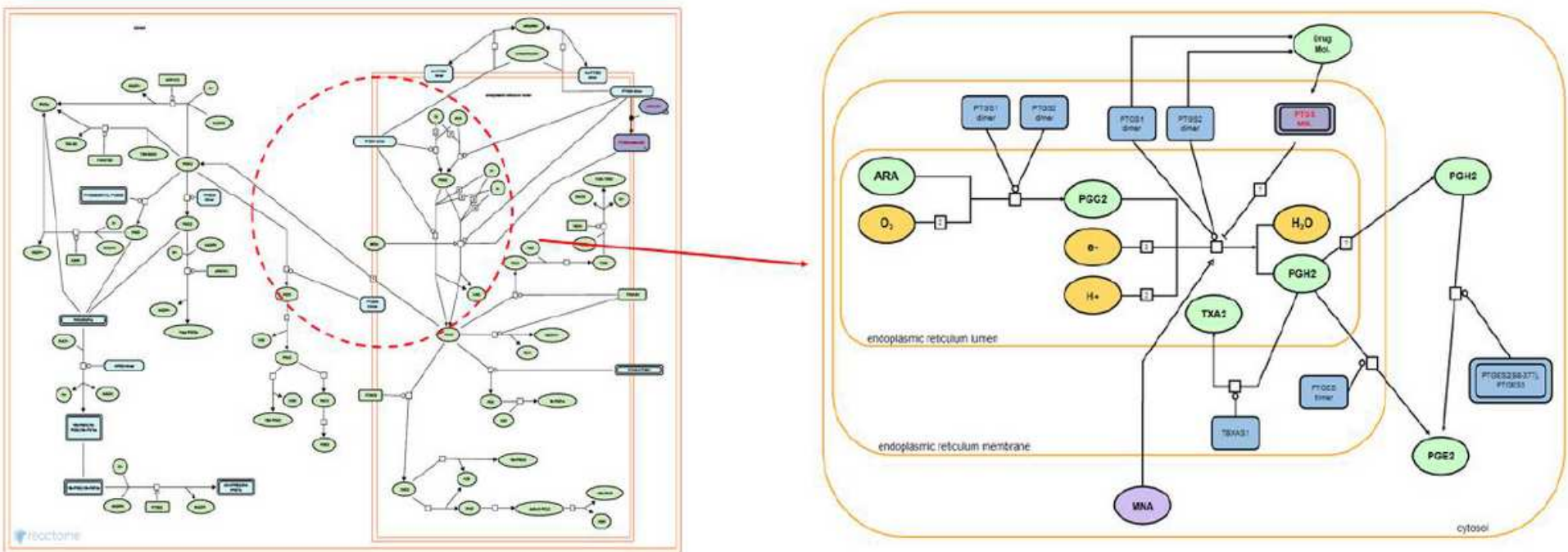

Figure 14

Arachidonic acid metabolism (R-HSA-2162123.2)

\section{Supplementary Files}

This is a list of supplementary files associated with this preprint. Click to download.

- DecoctionofOsteoarthritisoriginaldata.xlsx

- GeneCardsSearchResults.csv

- Graphicalabstracts.pdf

- OMIMEntryRetrieval.xIsx 